\title{
Influencia del medio ambiente en evaluación de stock: una aproximación con modelos globales de producción*
}

\author{
Pierre Fréon ${ }^{(1)}$ y Eleuterio Yáñez R. ${ }^{(2)}$ \\ (1) ORSTOM, BP 5045, 34032 Montpellier Cedex 1, France \\ (2) Escuela de Ciencias del Mar, UCV, Casilla 1020, Valparaíso, Chile
}

RESUMEN. Los modelos de producción convencionales no son adecuados para ciertos stocks, porque las variaciones del esfuerzo de pesca explican sólo una parte de la variabilidad total de las capturas anuales. A menudo la variación residual es originada por fenómenos ambientales, que afectan la abundancia y/o la capturabilidad del stock de un año a otro. En consecuencia, se ha incluido en los modelos convencionales una variable ambiental para mejorar la exactitud. Esta variable aparece en las fórmulas a nivel de la abundancia, de la capturabilidad, o de ambas. Estos modelos son desarrollados a partir del modelo de producción lineal de Schaefer, del exponencial de Fox o del generalizado de Pella y Tomlinson.

CLIMPROD es un sistema-experto experimental que proporciona una descripción estadística y gráfica de los datos y permite la selección del modelo correspondiente según los criterios objetivos del usuario. El software ajusta los modelos usando rutinas de regresión no-lineal, evalúa el ajuste a través de tests paramétricos y no-paramétricos, y proporciona una representación gráfica de los resultados.

Se consideran las limitaciones de este tipo de modelos. No obstante, éstos pueden proporcionar una interpretación bastante acertada de la historia de la pesquería, particularmente en el caso de stocks que colapsan inesperadamente sin ningún aumento apreciable del esfuerzo de pesca. Estos modelos también pueden ser útil en el manejo eficiente de este tipo de pesquerías, cuando los fenómenos climáticos pueden ser pronosticados o cuando su influencia esté restringida a la explotación del año(s) anterior. Finalmente se presenta el análisis de dos pesquerías colapsadas por una combinación de sobrepesca y cambios ambientales: la pesquería de sardina de Senegal y la de anchoveta del Pacífico suroriental.

Palabras claves: evaluación de stock, modelos de producción, manejo, software, ambiente, sardina, anchoveta.

\section{The influence of environment on stock assessment: an approach with surplus production models}

\begin{abstract}
Conventional global production models are not suitable for certain stocks, because fishing effort variations explain only a part of the total variability of annual catches. Often the residual variability originates from the influence of environmental phenomena, which affect either the abundance or the catchability of a stock from one year to the next. Therefore, an additional environmental variable has been inserted into conventional models in order to improve their aeeuracy. This variable appear in simple formulae concerning either stock abundance, or the catchability coefficient, or both. The models were developed from Schaefer's linear production model, Fox's exponential model or Pella and Tomlinson generalised model.

CLIMPROD is an experimental expert-system, using artificial intelligence, which provides a statistical and graphical description of the data set and helps the user to select the model corresponding to his case according to objective eriteria. The software fits the model to the data set using a non-linear regression routine, assesses the fit with parametric and non-parametric tests, and provides a graphical representation of the results.

Limitations of this kind of model are considered. The models can provide a fairly good interpretación of fishery history, particularly when a stock collapses unexpectedly without any appreciable increase in the nominal fishing effort. These models can also pro vide a useful tool efficient management of a fishery in those instances where climatic phenomena can be forecast, or when their influence is restricted to the year(s) preceding exploitation. Finally, the ana-
\end{abstract}

* Trabajo presentado en las X Jornadas en Pesquerías Chilenas, UCV, noviembre de 1992, Valparaíso. 
lysis of two fisheries collapsed by a combination of overfishing and environmental changes is presented: the Senegalese sardine fishery and the south-east Pacific anchovy fishery.

Key words: stock assessment, production models, management, software, environment, sardine, anchovy.

\section{INTRODUCCION}

Los modelos de excedente de producción convencionales empleados en evaluación de stock utilizan sólo una variable explicatoria, normalmente el esfuerzo de pesca. A partir del modelo lineal de Schaefer (Graham, 1935; Schaefer, 1954), se han desarrollado otros dos modelos globales que han sido ampliamente utilizados: el modelo exponencial (Garrod, 1969; Fox, 1970) y el modelo generalizado de producción (Pella y Tomlinson, 1969). Estos han sido desarrollados y adaptados para mejorar el ajuste de los datos observados, particularmente para condiciones de no equilibrio de la pesquería o para desfases del tiempo de respuesta del stock (Schaefer, 1957; Gulland, 1969; Fox, 1975; Walter, 1973, 1975, 1986; Schnute, 1977; Fletcher, 1978; Rivard y Bledsoe 1978; Uhler, 1980). En estos modelos, la variabilidad no asociada a la pesquería se considera como un ruido aleatorio, y algunos modelos estocásticos utilizan una variable aleatoria (Doubleday, 1976).

Aunque las relaciones entre las variaciones ambientales y la abundancia o disponibilidad de stocks han sido descritas anteriormente (Saville, 1980; Le Guen y Chevallier, 1983; Sharp y Csirke, 1983; Csirke y Sharp, 1983), el uso formal de modelos determinísticos que consideran el esfuerzo de pesca $\mathbf{E}$ y la variable ambiental $\mathbf{V}$ aparecen sólo a mediados de los ochenta (Fréon, 1983, 1984, 1988; Cury y Roy, 1989; Orbi et al., 1991; Yáñez, 1989, 1991; Yáñez et al., 1992). No obstante, semejante aproximación fue sugerida por Dickie (1973), mientras que Griffin et al. (1976) usaron una relación empírica entre el rendimiento de los camarones $\mathbf{Y}$ por una parte, y el esfuerzo de pesca $\mathbf{E}$ y el flujo del río $\mathbf{V}$ por la otra:

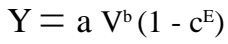

donde a, b y c son parámetros constantes. Esta relación es una función asintótica creciente y relevante sólo en algunos casos especiales. Sin embargo, las bases teóricas para tales modelos están disponibles en varias publicaciones sobre ecología acuática o terrestre. Algunos autores han introducido variables hidroclimáticas en los modelos estructurales de producción (Nelson et al., 1977; Loucks y Sutcliffe, 1978; Parrish y Mac Call, 1978), pero todos ellos requieren de datos detallados sobre la historia natural, tal como lo requieren algunos modelos de simulación complejos (Laevastu y Larkins, 1981).

Este trabajo entrega las bases teóricas sobre modelos de producción utilizando un factor ambiental como variable independiente, además del esfuerzo de pesca. La influencia del factor ambiental ha sido considerada a dos niveles: sobre la abundancia y sobre la capturabilidad del stock. Para cada caso, los modelos lineales y exponenciales (y algunas veces el modelo generalizado) son considerados. Luego se consideró el efecto simultáneo sobre la abundancia y la capturabilidad.

Se consideran también las limitaciones y aplicaciones de este tipo de modelos en estados transitorios (condiciones de no-equilibrio). Además se señalan las implicancias para el manejo de las pesquerías, en especial para los stocks inestables, y se describen los métodos y criterios de ajuste, así como la selección del modelo apropiado. Finalmente, se presenta el software CLIMPROD que permite desarrollar todas estas tareas y cómo superar algunas de ellas, así como dos ejemplos de aplicación asociados a pesquerías pelágicas.

\section{ACCION DE UNA VARIABLE AMBIENTAL EN MODELOS GLOBALES DE PRODUCCION}

\section{Definiciones}

Sea $\mathbf{V}$ una variable ambiental que representa cualquier factor probable de modificar las capturas de pesquerías; los ejemplos más comunes son: temperatura, salinidad, velocidad del viento, turbidez, magnitud y dirección de las corrientes, y flujo de los ríos.

En el trabajo se utilizará la notación convencional, principalmente la de Ricker (1975):

e: base de los logaritmos naturales 


\section{B: biomasa instantánea del stock \\ $\mathrm{B}_{\mathrm{i}}$ : $\quad$ biomasa promedio anual \\ $\mathrm{B}_{\infty}$ : biomasa máxima limitada por el ambiente o capacidad de carga ( $\mathrm{K}$ de los modelos ecológicos terrestres) \\ k: constante de la tasa de incremento de la población ( $\mathrm{r}$ de los modelos ecológicos terrestres) \\ $\mathrm{t}$ : tiempo, convencionalmente en años \\ F: mortalidad por pesca \\ q: $\quad$ coeficiente de capturabilidad \\ $E_{i}$ : esfuerzo de pesca anual durante el año $i$, estandarizado de modo que sea proporcio- nal a $F: F_{i}=q_{i} E_{i}$ \\ $Y_{i}: \quad$ rendimiento anual \\ $\mathrm{U}_{\mathrm{i}}$ : $\quad$ media anual de la captura por unidad de esfuerzo (CPUE) \\ $\mathrm{E}_{\max }$ : esfuerzo óptimo correspondiente al $\mathrm{Y}_{\max }$ \\ $\mathrm{Y}_{\max }$ : rendimiento máximo sostenido \\ $\mathrm{U}_{\max }$ : CPUE óptima correspondiente al $\mathrm{Y}_{\max }$ \\ $\mathrm{B}_{\mathrm{e}}, \mathrm{E}_{\mathrm{e}}, \mathrm{Y}_{\mathrm{e}}$ y $\mathrm{U}_{\mathrm{e}}$ : corresponden respectivamente a $\mathrm{B}$, $\mathrm{E}, \mathrm{Y}$ y $\mathrm{U}$ bajo condiciones de equilibrio}

\section{Antecedentes}

Los antecendentes y la forma de introducir una variable ambiental en los modelos se presentan sólo para el modelo lineal; más detalles sobre otros modelos se presentan en el trabajo de Fréon (1988).

Los modelos de excedente de producción se basan en la expresión de la ecuación logística, en términos de la tasa relativa de incremento del stock:

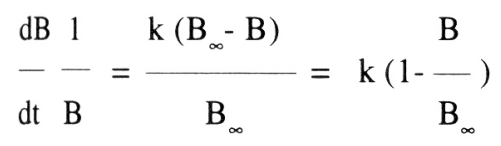

Varios autores (síntesis en Mac Call, 1984), trabajando en ecología terreste, estudiaron los efectos de las modificaciones del habitat (en tiempo o espacio) sobre esta relación. La modificación del habitat puede ser teóricamente introducida en la ecuación (1), de tres formas diferentes: efecto sólo sobre $\mathbf{B}_{\mathbf{o v}}$, efecto sólo sobre $\mathbf{k}$, y efectos sobre $\mathbf{B}_{\mathbf{~} 0}$ y $\mathbf{k}$. Habiendo analizado todos estos casos, Mac Call (1984) concluye que el último es el más conveniente, especialmente utilizando la solución de una pendiente constante para la ecuación (1):

$$
\frac{\mathrm{dB}}{\mathrm{dt}} \frac{1}{\mathrm{~B}}=\mathrm{k}-\mathrm{hB}
$$

donde $\mathbf{k}$ mantiene el mismo significado y $\mathbf{h}$ es la pendiente de la tasa relativa de incremento de la población. Esto significa que $\mathbf{h}=\mathbf{k} / \mathbf{B}_{\mathbf{o}}=$ constante y que $\mathbf{h}$ corresponde a $\mathbf{k}_{\mathbf{1}}$, de Schaefer (1954), quien también la considera como una constante.

Expresando la tasa absoluta de incremento del stock explotado como una función de la capacidad ambiental y la tasa de mortalidad por pesca como qf, conduce a la ecuación convencional del modelo de Schaefer:

$$
\frac{d B}{d t}=k B-h B^{2}-q E B=h B(B-B)-q E B
$$

\section{Introducción de una variable ambiental}

Utilizando esta formulación, los factores ambientales pueden interactuar sólo a dos niveles: con q si la capturabilidad está cambiando, o con el par de variables k-B $\mathbf{B}_{\mathbf{o}}$ (siendo la razón de estas dos variables una constante), si se considera la variablidad natural de la abundancia. En este último caso, para una presentación más fácil, se escogieron sólo aquellas fórmulas en que aparecen $\mathbf{B}_{\mathbf{w}} \mathbf{y} \mathbf{h}, \mathrm{Y}$ permitiendo que $\mathbf{B}_{\mathbf{o}}$ cambie de acuerdo al ambiente. Debe notarse, sin embargo, que cualquier variación de $\mathbf{B}_{\mathbf{o}}$ corresponde a una variación simétrica en $\mathbf{k}$. Más aún, $\mathbf{B}_{\mathbf{o v}}$ en las formulaciones matemáticas de los modelos de producción, no puede ser interpretada simplemente como la capacidad de carga para el stock reclutado. Otras evidencias (Sharp, 1980), indican que los procesos temporales y espaciales que afectan la dispersión de huevos y larvas pueden muy bien dominar los procesos energético/tróficos densodependientes en la limitación de la biomasa de la cohorte antes del reclutamiento. En tales casos, los stocks de adultos no necesariamente ocuparán la capacidad de carga de su ambiente.

Sea $\mathbf{g}(\mathbf{V})$ la función que representa las fluctuaciones de $\mathbf{B}_{\mathbf{o}}$ debidas a un factor ambiental representado por la variable $\mathbf{V}$, e $\mathbf{y}\left(\mathbf{V}^{\prime}\right)$ las fluctuaciones de $\mathbf{q}$ asociadas con otra variable ambiental $\mathbf{V}$ '. El modelo de Schaefer supone que, bajo condiciones 
de equilibrio, la tasa de incremento de la población es cero, lo que puede obtenerse de (3) si:

$$
B_{e}=B_{\infty}-q f / h=g(V)-y\left(V^{\prime}\right) f / h
$$

de modo que:

$$
\begin{aligned}
& U_{e}=q B_{e}=q B_{\infty}-q^{2} f / h=y\left(V^{\prime}\right) g(V)-y^{2}\left(V^{\prime}\right) f / h \\
& Y_{e}=f U_{e}=q B_{\infty} f-q^{2} f^{2} / h=y\left(V^{\prime}\right) g\left(V^{\prime}\right) f-y^{2}\left(V^{\prime}\right) f^{2} / h
\end{aligned}
$$

$\mathbf{f}_{\text {max }}$ será el valor de $\mathbf{f}$ obtenido anulando la derivada de la ecuación (6), tal que:

$$
\mathrm{f}_{\max }=\mathrm{B}_{\infty} \mathrm{h} / 2 \mathrm{q}=\mathrm{g}(\mathrm{V}) \mathrm{h} / 2 \mathrm{y}\left(\mathrm{V}^{\prime}\right)
$$

\section{Funciones $g(V)$ e $\mathbf{y}\left(V^{\prime}\right)$}

Las verdaderas funciones matemáticas de $\mathrm{g}(\mathrm{V})$ e $\mathrm{y}\left(\mathrm{V}^{\prime}\right)$, que enlazan una variable climática con $\mathbf{B}_{\mathrm{o}} \mathrm{y}$ q respectivamente, son por lo general desconocidas. Hasta aquí se ha utilizado una función muy flexible, expresada como:

$$
\mathrm{g}(\mathrm{V}) \text { ó } \mathrm{y}\left(\mathrm{V}^{\prime}\right)=\alpha+\beta \mathrm{V}^{\lambda} \text { ó } \alpha+\beta \mathrm{V}^{\prime \lambda}
$$

la que sólo será usada como una herramienta general en cuatro casos particulares, donde:

$$
\begin{array}{ll}
\alpha=0 ; \beta \neq 0 \text { y } \lambda=1 & \text { о́: bV } \\
\alpha=0 ; \beta=1 ; \lambda \neq 0 \text { y } \lambda \neq 1 & \text { о́: } \mathrm{V} \lambda \\
\alpha-0 ; \beta \neq 0 \text { y } \lambda=1 & \text { о́: } \alpha+\beta \mathrm{V} \\
\alpha=0 ; \beta \neq 0 \text { y } \lambda \neq 1 & \text { о́: } \beta \mathrm{V} \lambda
\end{array}
$$

Las funciones 8.1 y 8.1 I se justifican en casos particulares, es decir, cuando no se requiere una constante. La función $8.1 \mathrm{~V}$ es aún muy flexible, si sólo se está interesado en situaciones donde g(V) ó y(V') son funciones positivas y monotónicas, cubriendo un gran número de situaciones. Mac Call (citado por Fox, 1974), la usa para describir la relación entre q y $\mathbf{B}_{\mathrm{o}^{\circ}}$.

Cuando $\mathbf{g}(\mathbf{V})$ no es monotónica, sino una función con cierta forma, deben utilizarse otras ecuaciones, por ejemplo la función parabólica usada en este trabajo:

$$
\mathrm{g}(\mathrm{V}) \text { ó } \mathrm{y}(\mathrm{V})=\alpha \mathrm{V}-\beta \mathrm{V}^{2}
$$

El valor de los parámetros $\alpha, \beta$ y $\lambda$ (o el valor de los parámetros globales $\mathbf{a}, \mathbf{b}, \mathbf{c}$, ó $\mathbf{d}$ obtenidos después de reestructurar las ecuaciones), puede ser estimado ajustanto el modelo a los datos a través de la técnica de regresión. Los modelos con más de cuatro parámetros no serán utilizados, porque ellos reducen los grados de libertad en series de datos usualmente cortas.

\section{Modelos finales}

La línea de presentación utilizada hasta aquí sobre el modelo lineal o exponencial conduce a varios modelos, correspondientes al caso de la influencia del ambiente sobre la abundancia del stock (Fig. 1), la capturabilidad (Fig. 2), o sobre ambas (Figs. 3 y 4; Apéndice 1).

En la literatura se pueden encontrar numerosos ejemplos hipotéticos de la influencia ambiental sobre la abundancia, a través del reclutamiento y/o crecimiento poblacional (Blaxter y Hunter, 1982; Lasker, 1985), tales como: influencia de la intensidad de la surgencia, relaciones entre la producción del stock y la descarga de los ríos, influencia de la temperatura durante un estadio crítico, entre otros (Tabla 1). Esquemáticamente se han identificado cuatro períodos o estadios críticos:

- Antes del desove, influenciando la fecundidad del stock parental y/o el proceso de fecundación.

- Durante los primeros estadios de vida, influenciando la mortalidad natural de huevos y larvas (hambruna, comportamiento del depredador o abundancia).

- Durante el período de alta tasa de crecimiento (correspondiendo por lo general al estadio de pre-reclutamiento) cuando el ambiente influencia el crecimiento individual y/o la mortalidad natural (especialmente depredación).

- Durante el post-reclutamiento, donde la mortalidad natural y/o el factor de condición (y secundariamente la tasa de crecimiento) son de interés en este estadio.

Estos cuatro casos no son mutuamente excluyentes y en algunos casos es difícil identificar en qué estadio la influencia ambiental es mayor. No obstante, los estadios 1 a 3 (especialmente el estadio 2) se reconocen como los más importantes en términos de la variabilidad de la abundancia natural; mientras que el estadio 4 se refiere, generalmente, a la variabilidad de la mortalidad por pesca en relación 

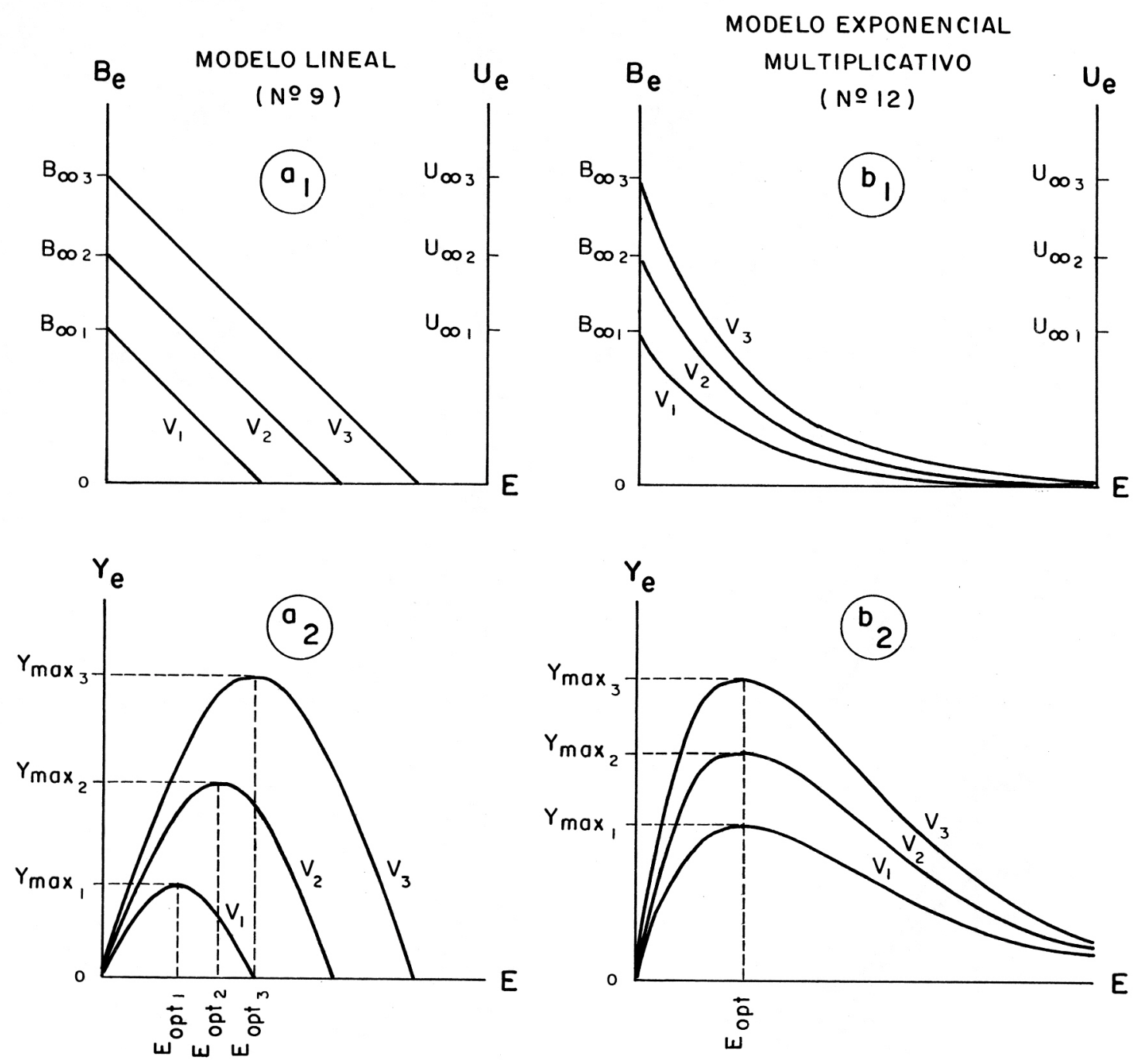

Figura 1. Modelo de producción lineal ( $a_{1}$ y $\left.a_{2}\right)$ y modelo exponencial multiplicativo $\left(b_{1}\right.$ y $\left.b_{2}\right)$, donde una varia ble ambiental $\mathrm{V}$ afecta la abundancia $\left(B_{o o}=g(V)\right)$ de acuerdo con tres valores diferentes $\left(V_{1}, V_{2}, V_{3}\right)$.

con los cambios ambientales.

El coeficiente de capturabilidad $\mathbf{q}$ puede ser asociado a las condiciones ambientales a través de cualesquiera de sus dos componentes: accesibilidad a la flota o vulnerabilidad al arte. Por ejemplo, los movimientos de las masas de agua pueden modificar los patrones migratorios y por lo tanto están asociados de la accesibilidad, especialmente en el caso de flotas de corto alcance. La turbidez del agua puede incrementar la vulnerabilidad del pez a algún tipo de arte (redes de enmalle, arrastre), o disminuida (pesca con luz). El caso en que q cambia de acuerdo a la abundancia del stock ha sido ya investigado por Fox (1974).

En algunos casos es razonable postular que el ambiente influencia a ambas, la abundancia del stock y la capturabilidad. En tales casos $\mathbf{B}_{\mathbf{0}}$ y $\mathbf{q}$ serán reemplazados por funciones de $\mathbf{V}$ y $\mathbf{V}$ ' respectivamente. En orden a limitar el número de parámetros y por lo tanto para evitar una gran disminución en los grados de libertad, se ha examinado sólo el caso simple donde la misma variable ambiental $\mathbf{V}$ influencia la abundancia y la capturabilidad $\left(\mathbf{V}=\mathbf{V}^{\prime}\right)$, y donde ambas $\mathbf{g}(\mathbf{V})$ e $\mathbf{y}(\mathbf{V})$ son descritas por la función (8.IV) 

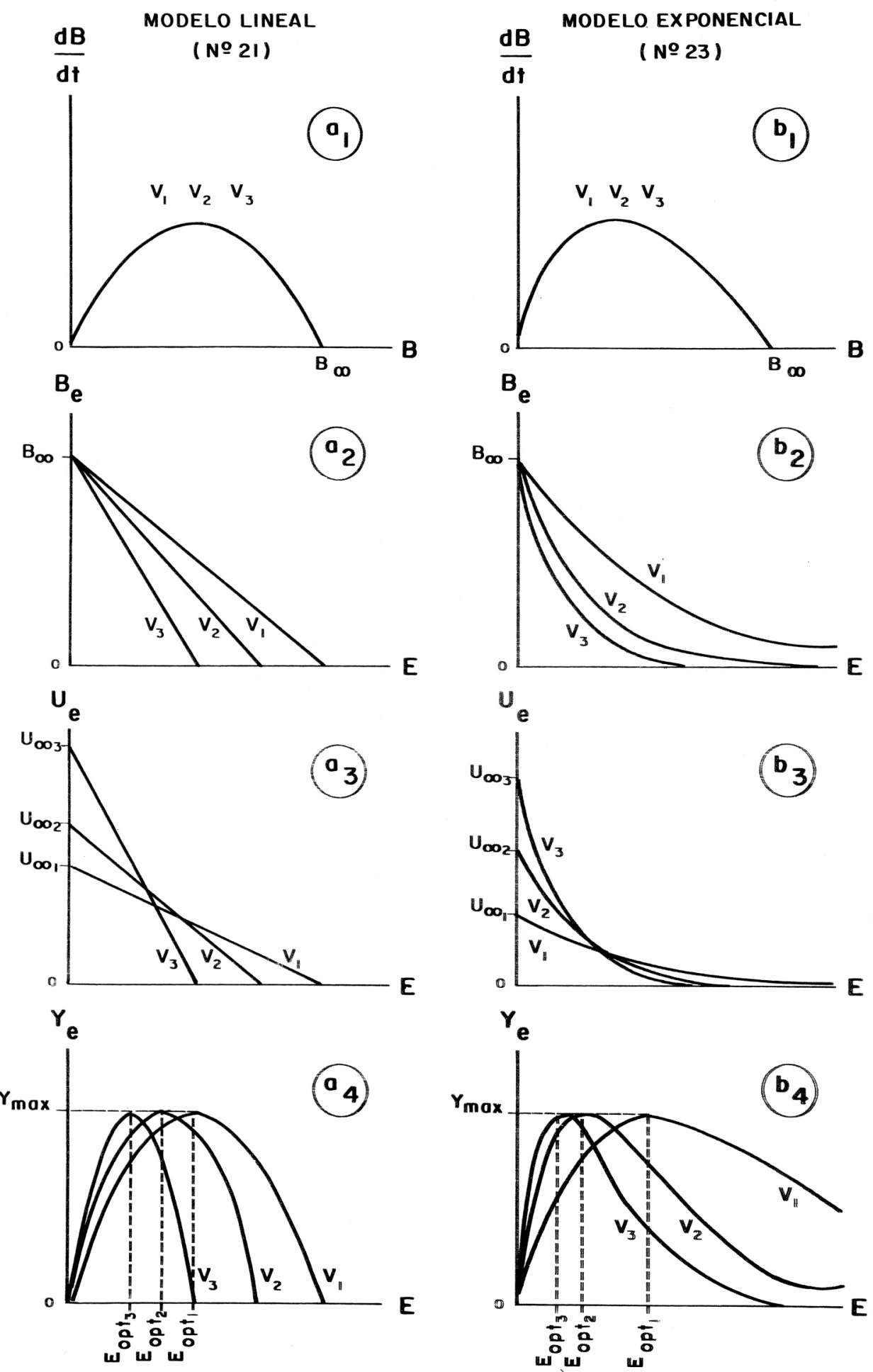

Figura 2. Modelo de producción lineaI $\left(a_{1} \ldots a_{4}\right)$ y modelo de producción aponendaI $\left(b_{1} \ldots b_{4}\right)$, donde una variable ambiental $V$ afecta la capturabitidad de acuerdo con tres valores diferentes $\left(V_{1}, V_{2}, V_{3}\right)$. 

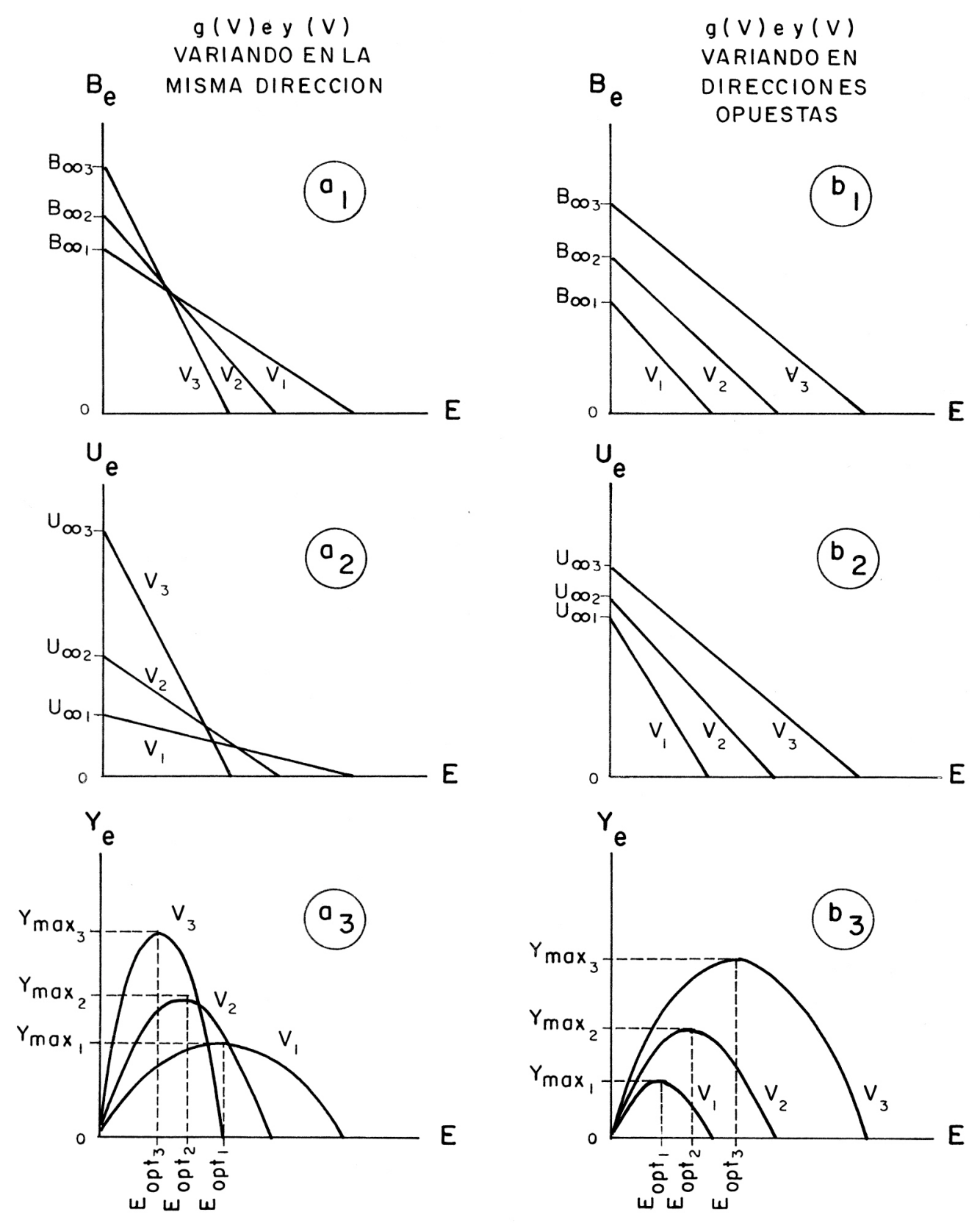

Figura 3. Modelo de producción lineal (\# 28) para tres valores de una variable ambiental $\mathbf{V}\left(\mathrm{V}_{1}, \mathrm{~V}_{2}, \mathrm{~V}_{3}\right)$ que afecta la abundancia del stock $\left(B_{o 0}=g(V)\right)$, y la capturabilidad $(q=y(V))$, cuando $g(V)$ e $y(V)$ varían en la misma dirección $\left(a_{1}, a_{2} y a_{3}\right)$ o en direcciones opuestas $\left(b_{1}, b_{2} y b_{3}\right)$. 

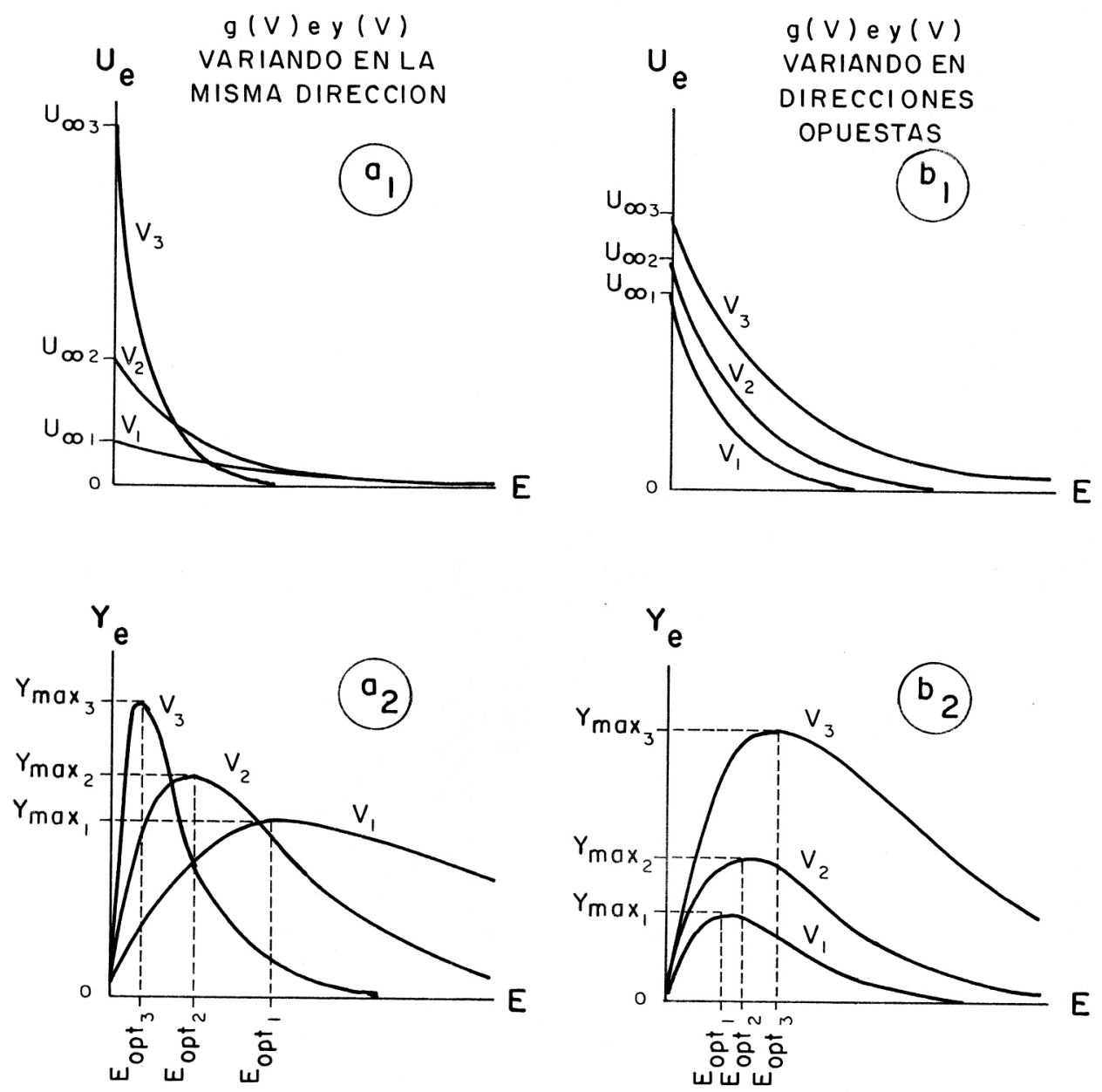

Figura 4. Modelo de producción exponencial (\# 30) para tres valores de una variable ambiental $\mathbf{V}\left(\mathbf{V}_{1}, \mathbf{V}_{2}, \mathbf{V}_{3}\right)$ que afecta la abundancia del stock $\left(B_{o o}=g(V)\right)$ y la capturabilidad $(q=y(V))$, cuando $g(V)$ e $y(V)$ varían en la misma dirección $\left(a_{1}\right.$ y $\left.a_{2}\right)$ o en direcciones opuestas $\left(b_{1}\right.$ y $\left.b_{2}\right)$.

(Apéndice 1; Figs. 3 y 4). Esto es aceptable porque esta función es muy flexible, pero teóricamente nada permite suponer que $\mathbf{g}(\mathbf{V})$ e $\mathbf{y}(\mathbf{V})$ serán idénticas. Más aún, la aproximación utilizada para estimar los parámetros del modelo en el caso de estados transicionales, a través del promedio de esfuerzos pasados, permite el uso de estos modelos sólo en casos particulares (véase más abajo). Un ejemplo donde una variable ambiental (temperatura superficial del mar) influencia la abundancia y otra (descarga del río) la capturabilidad es dado por Fréon (1988).

\section{USO DE MODELOS CON DATOS EN ESTADOS DE TRANSICION}

\section{Presentación general}

Las ecuaciones precedentes se basan en un stock en estado de equilibrio a varios niveles estables de esfuerzo de pesca y condiciones ambientales. La aproximación de predicción en situación de transición fue adoptada para ajustar un modelo a los datos observados. Esta consiste en ajustar los datos de esfuerzo de pesca y ambiente en orden a estimar un estado de equilibrio. Fox (1974) propone un promedio ponderado de las series de esfuerzo, en lugar del promedio 
Tabla 1. Efectos ambientales sobre modelos de producción: variables claves, mecanismos biológicos involucrados, períodos de tiempo de los efectos, desfaces sobre la producción, tipos de efectos y signos.

\begin{tabular}{|c|c|c|c|c|c|c|}
\hline $\begin{array}{l}\text { MECANISMO } \\
\text { AMBIENTAL }\end{array}$ & $\begin{array}{l}\text { VARIABLE } \\
\text { CLAVE }\end{array}$ & $\begin{array}{l}\text { MECANISMO } \\
\text { BIOLOGICO }\end{array}$ & $\begin{array}{l}\text { PERIODO } \\
\text { DE TIEMPO }\end{array}$ & $\begin{array}{c}\text { DESFASE } \\
\text { PRODUCCION }\end{array}$ & $\begin{array}{c}\text { EFECTO } \\
\text { SOBRE }\end{array}$ & SIGNO \\
\hline $\begin{array}{l}\text { Aumento de produc- } \\
\text { ción primaria por sur- } \\
\text { gencia }\end{array}$ & $\begin{array}{l}\text { Velocidad del viento o } \\
\text { transporte Ekman o } \\
\text { TSM }\end{array}$ & Fecundidad & Estación & Años & Abundancia & + \\
\hline " & " $"$ & $\begin{array}{l}\text { Mortalidad natural } \\
\text { (cualquier estadio) }\end{array}$ & Estación o año & Años & Abundancia & + \\
\hline$"$ & $"$ & Crecimiento & \begin{tabular}{|l} 
Estación o año \\
\end{tabular} & Años & Abundancia & + \\
\hline$"$ & $"$ & Patrón migratorio & Estación & Mes & Capturabilidad & + \\
\hline " & $"$ & Nivel de agregación & Estación & \begin{tabular}{|l|} 
Sin desfase \\
o días \\
\end{tabular} & Capturabilidad & $+o ́-$ \\
\hline $\begin{array}{l}\text { Aumento de corrientes } \\
\text { (surgencia u otros) }\end{array}$ & $\begin{array}{l}\text { Transporte Ekman } \\
\text { o datos de corrientes }\end{array}$ & Advección larval & Mes & Años & Abundancia & - \\
\hline $\begin{array}{l}\text { Turbulencia en la co- } \\
\text { lumna de agua }\end{array}$ & $\begin{array}{l}\text { Velocidad del viento } \\
\text { al cubo }\end{array}$ & \begin{tabular}{|l|} 
Mortalidad natural \\
de larvas (disponibi- \\
lidad de alimento) \\
\end{tabular} & Mes & Años & Abundancia & - \\
\hline $\begin{array}{l}\text { Anomalías de la tem- } \\
\text { peratura del mar (no } \\
\text { ligadas a surgencia) }\end{array}$ & $\begin{array}{l}\text { TSM o temperatura } \\
\text { de la columna de } \\
\text { agua o profundidad } \\
\text { de la termoclina }\end{array}$ & $\begin{array}{l}\text { Mortalidad natural } \\
\text { (efecto físico sobre } \\
\text { sobrevivencia de } \\
\text { huevos y larvas) } \\
\end{array}$ & Mes & Años & Abundancia & $+\dot{o}-$ \\
\hline$"$ & " & Cambio en bio-topo & Año & Años & Abundancia & $+\dot{0}-$ \\
\hline$"$ & $"$ & $\begin{array}{l}\text { Cambio en distribu- } \\
\text { ción vertical u hori- } \\
\text { zontal, o en agrega- } \\
\text { ción }\end{array}$ & Estación o año & $\begin{array}{l}\text { Sin desfase } \\
\text { o días }\end{array}$ & Capturabilidad & $+\dot{o}-$ \\
\hline $\begin{array}{l}\text { Aumento de produc- } \\
\text { ción primaria por des- } \\
\text { carga de ríos }\end{array}$ & $\begin{array}{l}\text { Flujo del río o nivel } \\
\text { cerca de la boca o } \\
\text { extensión de la plu- } \\
\text { ma (observación sa- } \\
\text { telital) o lluvia }\end{array}$ & $\begin{array}{l}\text { Fecundidad } \\
\text { (cualquier estadio) }\end{array}$ & Estación o año & Años & Abundancia & + \\
\hline " & " $"$ & \begin{tabular}{|l|} 
Mortalidad natural \\
(cualquier estadio)
\end{tabular} & Estación o año & Años & Abundancia & + \\
\hline " $\quad "$ & " " & \begin{tabular}{|l|} 
Crecimiento \\
\end{tabular} & Estación o año & Años & Abundancia & + \\
\hline $\begin{array}{l}\text { Cambios fisicos de ma- } \\
\text { sas de agua en relación } \\
\text { con descarga de los ríos }\end{array}$ & $\begin{array}{l}\text { o salinidad } \\
\text { o turbidez }\end{array}$ & $\begin{array}{l}\text { Distribución es- } \\
\text { pacial o nivel de } \\
\text { agregación }\end{array}$ & Estación o año & $\begin{array}{l}\text { Sin desfase } \\
\text { o días }\end{array}$ & Capturabilidad & $+o ́-$ \\
\hline
\end{tabular}

TSM: temperatura superficial del mar

simple inicialmente sugerida por Gulland (1969). La misma aproximación puede ser utilizada para la variable ambiental (Fréon, 1988).

Esta aproximación es fácil de usar, pero una de las caras del problema es el artificio causado por la no-independencia de las series de datos del esfuerzo de pesca y de la CPUE (Roff y Fairbairn, 1980). Esta aproximación no es precisa cuando $\mathbf{g}(\mathbf{V})$ y/o $\mathbf{y}(\mathbf{V})$ no son funciones lineales, ni aceptable en el caso de funciones nomonotónicas cuando la variación interanual de $\mathbf{V}$ es grande y cuando, por algunos años, el valor promedio de $\mathbf{V}$ resulta de valores ubicados a cada lado del valor óptimo. No obstante, se propone adaptar a los modelos de producción ambiental por razones pragmáticas. Se reconoce que la aproximación de predicción en situación de transición puede conducir a un cierto sesgo o errores que afectan la estimación de los parámetros, como ha sido enfatizado por Walter (1975), Schnute (1977), Uhler (1980) y Hilborn y Walters (1992). Sin embargo, las mejores estimaciones estadísticas de los parámetros no necesariamente proveen las mejores estimaciones de $\mathbf{Y}_{\text {max }}$ y $\mathbf{F}_{\text {max }}$, que son el objetivo principal de los modelos de producción global (Uhler, 1980). 


\section{Estados de transición e influencia ambiental}

Con respecto a la variable ambiental, el uso de la aproximación de predicción en transición supone que la etapa de vida durante la cual el ambiente actúa sobre el stock es conocida. Cuanto más corto el período de vida (o al menos la etapa de vida capturable), tanto mejor será la aproximación. En tales casos es más fácil determinar y cuantificar el efecto ambiental sobre la capturabilidad o sobre la abundancia. En el último caso, las situaciones más favorables son provistas por rápida acción del ambiente sobre un estadio de vida, o por fluctuaciones menores del ambiente (series de datos autocorrelacionados). Con el propósito de simplificar la presentación, las fluctuaciones interanuales del ambiente pueden considerarse como cíclicas, con un período T. Sin embargo, en la mayoría de los casos, la realidad no es más que una alternancia entre anomalías climáticas positivas y negativas, no necesariamente de la misma duración. En los casos especiales donde las fluctuaciones ambientales son verdaderamente periódicas, podrían observarse las frecuencias resonantes del ecosistema, según lo hace notar Silvert (1983).

Si las series de datos de la pesquería tienen una duración despreciable comparadas con $\mathbf{T}$ (una escala de centurias por ejemplo), sería difícil cuantificar una eventual influencia ambiental tal como lo sugieren los resultados de Soutar y Isaacs (1974). Los modelos estocásticos de producción que usan una función periódica también pueden usarse en tal caso (Steele y Henderson, 1984). Cuando la extensión de las series de datos de la pesquería es más corta que $\mathbf{T}$ pero más grande que $\mathbf{T} / \mathbf{4}$, se puede intentar un modelo si, por casualidad, toda la serie de datos se ubica sobre un solo lado (aumentando o disminuyendo) de la función periódica T. Pero en este caso limitado, cualquier extrapolación de los resultados sería peligrosa.

Si la duración del estadio crítico $\mathbf{p}$ es mayor ó igual a T, será muy difícil identificar los efectos ambientales porque serán suavizados para cada cohorte. Las condiciones más favorables parar usar estos modelos ocurren cuando $\mathbf{p}$ es menor que $\mathbf{T}$, ,especialmente cuando es menor que $\mathbf{T} / \mathbf{2}$, y cuando la duración de la etapa de vida capturable n también es más corta que T/2. En tales casos, la mezcla de varias cohortes en capturas anuales producirán un suavizamiento mínimo de los rendimientos globales.

\section{IMPLICANCIAS EN EL MANEJO DE PESQUERIAS}

\section{Influencia del ambiente sobre la abundancia}

Se ha visto que, en algunos casos, los efectos ambientales sobre la abundancia del stock es algo más que sólo ruido y por lo tanto es posible modular el esfuerzo de pesca de acuerdo a las predicciones de abundancia.

En situaciones donde la previsión de la abundancia es con fiable (desface de la influencia climática o conecciones remotas), la dificultad del manejo resultará de su objectivo dual: optimización del rendimiento con incrementos del esfuerzo cuando la abundancia aumenta y protección del stock contra el colapso con rápidas reducciones del esfuerzo de pesca cuando los factores ambientales son desfavorables. Tal colapso puede, de hecho, suceder rápidamente sin ningún aumento del esfuerzo si se mantiene un esfuerzo óptimo, que ya no corresponde a la condición climática actual (Fig. 5a). El colapso ocurrirá más rápidamente cuando pocas cohortes explotadas actúan como buffer y cuando el estadio de vida crítico dura menos de un año (Fréon, 1983 y 1984). Este ajuste permanente del esfuerzo de pesca no es fácil de aplicar, porque hay un desfase entre las ganancias y las inversiones. Un análisis de este problema está disponible en Csirke y Sharp (1983). El manejo pesquero puede basarse en cuotas anuales variables o en esfuerzos máximos variables permisibles.

\section{Influencia del ambiente sobre la capturabilidad}

Aquí el principal riesgo de colapso ocurre cuando se cambia desde condiciones climáticas favorables a condiciones climáticas desfavorables. Siguiendo el modelo bio-económico usual (Troadec, 1982), la primera situación conducirá luego de unos pocos años a un aumento del esfuerzo de pesca asociado a capturas cercanas a $\mathbf{Y}_{\text {max }}$ (Fig. 5b). Entonces, cuando la capturabilidad aumenta repentinamente, también pueden aumentar los rendimientos y el estado de no equilibrio de la pesquería resultará en su colapso. En tal caso, la decisión de manejo más apropiada sería la de fijar una sola cuota, generalmente más fácil de determinar y de controlar que las limitaciones del esfuerzo variable. 

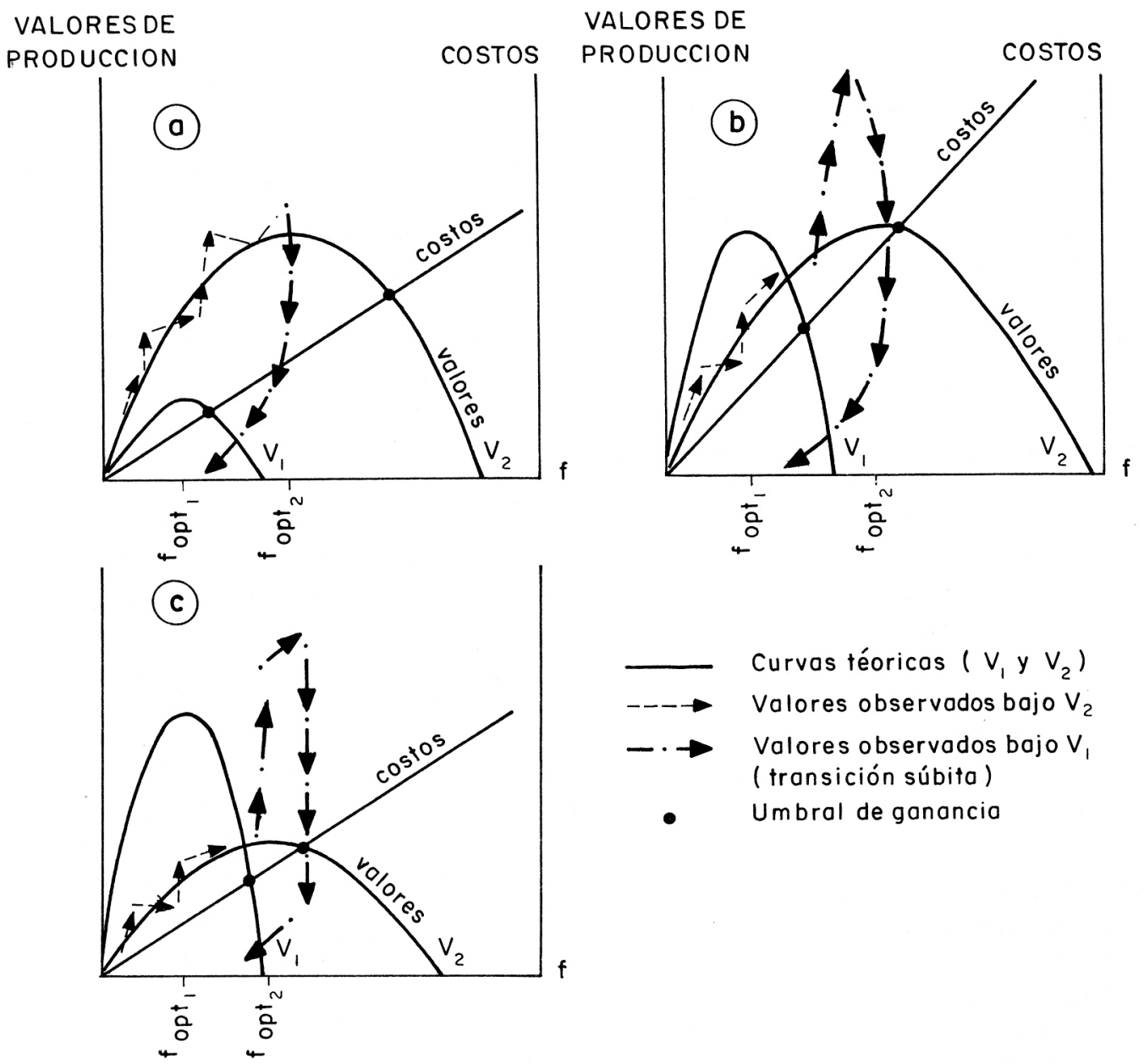

Figura 5. Modelo de producción bio-económico y ejemplos teóricos de colapsos de stocks cuando el ambiente afecta la abundancia del stock (a), la capturabilidad (b) o ambos factores en la misma dirección (c), de acuerdo con dos valores de la variable ambiental $\mathrm{V}\left(\mathrm{V}_{1}, \mathrm{~V}_{2}\right)$.

\section{Influencia del ambiente sobre la abundancia y la capturabilidad}

Dependiendo de si el ambiente influye sobre $\mathbf{B}_{\mathbf{~}}$ y $\mathbf{q}$ en la misma dirección o en dirección opuesta, las cifras resultantes serán completamente diferentes (Figs. 3a y 3b). Sólo dos casos extremos serán analizados aquí, pero existen numerosas situaciones intermedias.

En el primer caso, donde $\mathbf{g}(\mathbf{V})$ e $\mathbf{y}(\mathbf{V})$ tienen el mismo signo de variación, la súbita ocurrencia de un ambiente desfavorable relativo a la abundancia no es peligrosa porque la capturabilidad será baja. Por otra parte, cuando las condiciones ambientales son favorables para la abundancia y la capturabilidad, y si no hay mecanisnos de regulación previstos para una eventual saturación del mercado o ajustes de precios, el esfuerzo de pesca tendrá una tendencia a exceder $\mathbf{f}_{\max }$ (Fig. 5c). Se requiere entonces de una fuerte limitación.

En el segundo caso, cuando una alta abundancia está asociada con una baja capturabilidad, el principal riesgo de colapso ocurre cuando las malas condiciones climáticas siguen a las buenas. Esta situación es comparable a aquella descrita anteriormente para el caso de la influencia del ambiente sólo sobre la abundancia (Fig. 5a). 


\section{METODO Y CRITERIOS DE AJUSTE}

La mayor parte de estos modelos requieren regresión no-lineal para ajustarse, por ejemplo, aquellos basados en el algoritmo de Marquardt (1963), en el método modificado de Gauss-Newton (Dixon y Brown, 1979), o en el método Simplex (Nelder y Mead, 1965). Estos métodos son iterativos y usan el criterio de mínimos cuadrados. El ajuste puede hacerse por medio de fórmulas de $\operatorname{CPUE}\left(\mathbf{U}_{\mathrm{i}}\right)$ o capturas $\left(\mathbf{Y}_{\mathbf{i}}\right)$. Esta última solución hace el ajuste más difícil pero, teóricamente, evita el sesgo en los coeficientes de regresión debido a la no-independencia de $\mathbf{f}_{i}$ y $\mathbf{U}_{i}$ (dado que $\mathbf{f}_{i}$ y $\mathbf{Y}_{i}$ han sido estimados independientemente ).

Se pueden hacer modificaciones al procedimiento ponderando los residuales. Fox (1971) analizóeste problema y retuvo la solución considerando el error proporcional a las capturas estimadas $\mathbf{Y}_{\text {, }}$ lo que conduce a la minimización de la función $\mathbf{S}$ :

$$
\underset{i=1}{S^{n}}=\sum\left[\left(Y_{i}-Y_{i}\right) / Y_{i}\right]^{2}
$$

Todos los algoritmos necesitan de la estimación de valores de partida de los parámetros para inicializar el proceso iterativo. Con el objeto de evitar convergencias hacia mínimos locales o hacia soluciones irracionales desde el punto de vista biológico, los valores de partida deben ser estimados cuidadosamente. Esto puede hacerse utilizando la formulación inicial del modelo donde aparecen $\mathbf{B}_{\mathbf{0}} \circ \mathbf{U}_{\mathbf{o}}$. Sus valores pueden ser estimados duplicando la captura máxima (o CPUE) observada en las series de datos. Los exponentes $\mathbf{g}(\mathbf{V})$ e $\mathbf{y}(\mathbf{V})$ de la función pueden ser inicializados como I ó O.

Se puede obtener una estimación no-paramétrica del ajuste usando el método jack-knife o el de validación-cruzada (Ducan, 1978; Efron y Gong, 1983). Estos métodos muestran la estabilidad del modelo cuando se saca un año de observaciones de la serie de datos. Es interesante observar que, en algunos casos, todos los valores de los parámetros cambian mientras que el ajuste permanece más o menos igual dentro del rango de los datos observados, pero las curvas son divergentes fuera de este rango. Esto indica el riesgo de usar tales modelos fuera del rango de datos observados del esfuerzo de pesca y de los factores ambientales. En algunas ocasiones parece preferible fijar un valor razonable a uno de los parámetros, como ya ha sido mencionado por Pella y
Tomlinson (1969) en su modelo generalizado.

\section{SELECCION DEL MODELO APROPIADO}

Debido al número generalmente bajo de observaciones anuales y al número relativamente alto de parámetros a estimar, los modelos presentan pocos grados de libertad. Consecuentemente, la selección del modelo apropiado, de entre los numerosos modelos aquí presentados, no debe basarse razonablemente sobre el criterio del mejor ajuste. Información adicional, independiente de las series estadísticas de captura y esfuerzo, debe tomarse en cuenta a fin de evitar correlaciones sin sentido.

Es posible identificar dos categorías de criterios objetivos y se presentan aquí brevemente. Primero, debe decidirse si el ambiente influencia la abundancia del stock o la capturabilidad. La selección de modelos donde ambos fenómenos son considerados debe estar sustentada por observaciones, en lugar de ser una selección oportunística. El análisis de series de tiempo, utilizando un corto intervalo de tiempo puede permitir la distinción entre el efecto contemporáneo del ambiente sobre la capturabilidad y un efecto retardado sobre la abundancia (en este último caso, se puede estimar el desfase). Para extraer los efectos estacionales y determinar el estadio crítico, se pueden ejecutar funciones de transferencia entre CPUE y el ambiental. En aquellas instancias donde grandes variaciones interanuales del ambiente permiten detectar un efecto de largo plazo a pesar de un efecto estacional, puede ser muy útil entrevistar a los pescadores. El segundo paso es decidir si se debe usar un modelo de tipo lineal o exponencial, o el generalizado. Si el stock no ha sido nunca sobre-explotado, al sobrepasar los esfuerzos óptimos los tres tipos de modelo proveen ajustes similares. Sin embargo, las curvas son divergentes sobre aquellos esfuerzos máximos y es preferible dar una tendencia representativa, aunque no debiera usarse cualquier modelo para predecir situaciones fuera del rango observado de datos.

Información adicional sobre la estructura del stock puede ayudar a escoger entre un modelo lineal pesimista y uno exponencial que permite una lenta declinación hacia el colapso del stock. Datos cualitativos sobre la historia del stock pueden proveer directamente la información decisiva cuando ya se han reportado colapsos. Los modelos lineales son adecua- 
dos para especies de vida corta cuando todas las clases anuales son explotadas. La subdivision en sub-stocks, reservas naturales, donde la pesca es imposible o una alta selectividad del arte sobre los adultos incitaría a usar modelos exponenciales. El sistema-experto CLIMPROD optimiza la selección de acuerdo a tal información.

\section{SOFTWARE CLIMPROD}

\section{Presentación general}

El sistema-experto CLIMPROD hace uso de inteligencia artificial para escoger el modelo mejor adaptado a cada situación, y para evaluar el ajuste de acuerdo a las series de datos y al conocimiento que se tiene sobre el stock (Fréon et al., 1993). El software está escrito para micro-computadores PC/ $\mathrm{XT} / \mathrm{AT}$ compatibles que usan MS-DOS versión 3.0 (o superior). Este es completamente interactivo y tiene dos objetivos principales: en primer lugar una función normal de manejo de datos, cuyas utilidades estadísticas y gráficas usan lenguaje TURBO C; y en segundo lugar una selección guiada del modelo apropiado, que muestra los canales de información. Esta parte del modelo usa un ingenio inferencial, escrito en TURBO PROLOG. Este aplica cerca de cien reglas que son interactivas con la información provista por:

- preguntas al usuario sobre el stock, independientes del set de datos (por ejemplo: la duración de vida de las especies);

- estadísticas del set de datos (por ejemplo: la razón del rango del esfuerzo sobre el valor mínimo del esfuerzo); $\mathrm{y}$

- deducción gráfica del set de datos (por ejemplo: ¿Se ve inestable esta serie de tiempo?, ¿Ve Ud. una relación decreciente en este gráfico?).

La respuesta no lo sé si está permitida. El programa es estructurado y no necesariamente usa el conjunto completo de preguntas. Un ejemplo del orden en la aplicación de las reglas se presenta en la Fig. 6.

Desde el menú principal, se permite al usuario abrir o seleccionar un archivo de datos; modificarlo con un editor en pantalla completa; buscar el modelo más adecuado, o escoger uno directamente; validar el modelo (evaluar el ajuste); graficar la función modelo; predecir valores y residuales; ver los canales de las decisiones del experto; y finalmente utilizar el modelo para predecir.

Se hace notar que para escoger de entre 30 modelos multivariados (Apéndice 1), el programa realiza primero una regresión usando la CPUE como variable dependiente y el esfuerzo (o el ambiente en algunos casos) como variable independiente. De la presentación gráfica de los residuales de esta regresión versus la variable ambiental, el usuario puede determinar qué tipo de relación asociará el ambiente y la CPUE en el modelo multivariado final. Este procedimiento provee una fácil interpretación y visualización del proceso para seleccionar el modelo y permite el diálogo interactivo con el usuario, quien puede hacer uso de información adicional. No obstante, técnicas estadísticas recientes de transformación óptima para regresión múltiple (Breiman y Friedman, 1985; Cury y Roy, 1989) pueden ser más poderosas y óptimas para escoger el modelo desde un punto de vista estrictamente estadístico. Como estas técnicas sólo usan series de tiempo multivariada (la que a menudo es demasiado corta para su uso óptimo), debieran ser una ayuda complementaria útil en la selección del modelo.

\section{Ingreso y modificación de datos}

El set básico de datos usado por CLIMPROD incluye series de tiempo de captura $(\mathbf{Y})$, esfuerzo de pesca $(\mathbf{E}), \operatorname{CPUE}(\mathbf{U}=\mathbf{Y} / \mathbf{E})$, y de la variable ambiental (V). Un editor en pantalla completa permite el ingreso de datos, su corrección y actualización.

\section{Estadística univariada y gráficos de los datos crudos}

A cada variable se le calculan los siguientes estadísticos: tamaño muestral, promedio, varianza, desviación estándar, coeficiente de variación, coeficiente de sesgo y curtosis, valores mínimo y máximo, rango y mediana. La distribución de los datos se muestra en un histograma de frecuencia, que permite detectar valores extremos eventuales. Aun cuando ningún set de datos de pesquerías podría usarse si la normalidad fuese estrictamente requerida para modelar, estos resultados pueden dar una idea de la estructura de los datos. CLIMPROD detiene el análisis, y/o despliega un aviso o advertencia, de acuerdo a la distribución de los valores de las diferentes variables. Por ejemplo, el programa se detendrá si hay menos de 12 años de observación disponibles, o 


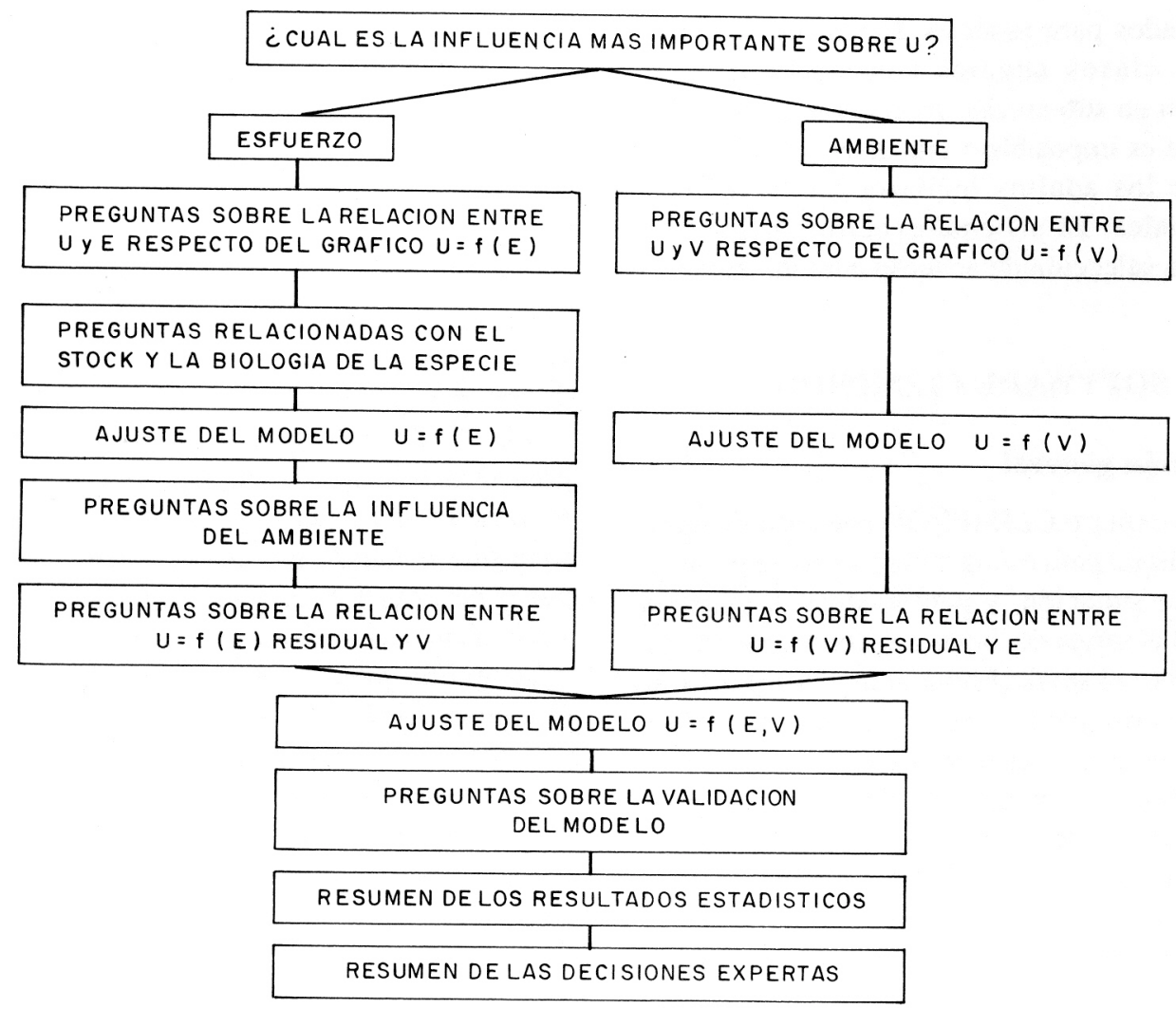

Figura 6. Diagrama de flujo parcial y simplificado de CLIMPROD, donde U es la captura por unidad de esfuerzo, $\mathrm{E}$ el esfuerzo de pesca y $\mathrm{V}$ una variable ambiental.

si la razón rango del esfuerzo/mínimo esfuerzo está bajo el $40 \%$.

\section{Examen de las series de tiempo}

Cada variable se grafica contra el tiempo (años) para detectar cualquier fuerte inestabilidad en las series, las que algunas veces entorpecen la interpretación de los resultados. En el caso de fuertes instabilidades de E o V por ejemplo, si el modelo retenido requiere promediar una de estas variables sobre varios años para aproximarse a un estado de equilibrio, los resultados serán de poco valor.

\section{Gráficos bivariados}

Se grafican las siguientes relaciones: $\mathbf{Y}$ versus $\mathbf{E}, \mathbf{Y}$ versus $\mathbf{V}$, $\mathbf{U}$ versus $\mathbf{E}$, $\mathbf{U}$ versus $\mathbf{V}$ y $\mathbf{V}$ versus $\mathbf{E}$. Estos gráficos revelan cualquier punto extremo que pueda estructurar el set de datos, o cualquier fuerte relación (lineal o no) entre las dos variables inde- pendientes $\mathbf{E}$ y $\mathbf{V}$. Debe subrayarse que actualmente el programa no considera los efectos de des fases potenciales entre las variables en esta etapa gráfica.

\section{Preguntas para guiar la selección del modelo}

Cuatro preguntas sobre supuestos básicos de los modelos de excedentes de producción se hacen en formas sistemática, y el programa se detiene si estos supuestos no se cumplen. Las siguientes preguntas se hacen también en forma sistemática:

- ¿Cree Ud. que la influencia del esfuerzo sobre la CPUE es más importante que la influencia del ambiente (si se desconoce, se asume que sí)? La respuesta, guiada por ayuda estadística y gráfica, orienta al programa primero hacia los modelos $\mathbf{U}=\mathrm{f}(\mathbf{E}) \mathrm{o}$ $\mathbf{U}=\mathrm{f}(\mathbf{V})$.

- ¿El ambiente influencia la abundancia, la capturabilidad o ambas? En este momento el programa no provee ninguna ayuda para responder es 
tas preguntas; se supone que el usuario conoce el mecanismo de acción del ambiente sobre el stock.

Entre estas dos preguntas, el programa hará una o varias preguntas en orden a determinar cuál relación es la más adecuada entre $\mathbf{U}$ y $\mathbf{E}$ (modelo lineal de Schaefer, modelo exponencial de Fox y Garrod, o el modelo generalizado de Pella y Tomlinson), y entre $\mathbf{U}$ y $\mathbf{V}$ (lineal, exponencial, general o cuadrático ).

\section{Ajuste del modelo}

En caso de condiciones de no-equilibrio (situaciones de transición), se calcula el promedio ponderado de $\mathbf{E}$ y/o V. En caso de influencia retardada del ambiente sobre la abundancia, se inserta un retraso entre el promedio ponderado de $\mathbf{V}$ y $\mathbf{U}$ (Fréon, 1988). El algoritmo de Marquardt se usa para la estimación de mínimos cuadrados de parámetros no lineales. De acuerdo al modelo, los valores iniciales de los parámetros son 1, 0 ó se calculan desde el set de datos originales antes de correr el algoritmo. Como primer resultado se da el porcentaje de variación explicado por el modelo $\left(\mathrm{R}^{2}\right)$. El paso siguiente depende de la calidad del ajuste, esto es:

- después del paso de estimar el modelo bivariado, si $\mathrm{R}^{2}<40 \%$ el programa para o invita al usuario a dar nuevas respuestas a las preguntas ya formuladas; si $\mathrm{R}^{2}>90 \%$ se puede intentar la validación del modelo bivariado. Si $40<\mathrm{R}^{2}<90 \%$, el programa intentará encontrar un modelo multivariado $\mathbf{U}=\mathrm{f}(\mathbf{E}, \mathbf{V})$ que provea una mejor correlación que el modelo bivariado.

- luego de la estimación del modelo multivariado, es posible intentar la validación si $\mathrm{R}^{2}>70 \%$.

\section{Prueba estadística de robustez (validación)}

La evaluación del ajuste está basado principalmente en una estimación jack-knife de los parámetros y de $\mathrm{R}^{2}$. También toma en cuenta el análisis de los residuales y las características del set de datos. De la presentación gráfica de los valores ajustados por el modelo y de sus residuales., se requiere finalmente la opinión del usuario.

\section{Resumen de la decisión experta}

Al término de cada paso del menú principal, el usuario puede desplegar los pasos seguidos por el programa en cada nivel de decisión con el número correspondiente de la norma.

\section{Ejemplos de aplicación}

Pesquería de sardina de Senegal

Se presenta un ejemplo de aplicación que corresponde a la pesquería de sardina de Senegal (Fréon, 1983; 1988). Se usa tentativamente un nuevo índice de abundancia, el peso promedio anual por lance cuando se realiza un único lance exitoso por viaje (Fréon, 1991a). La variable ambiental que influencia la abundancia del stock es la media de la velocidad del viento durante la época de surgencia.

De acuerdo al conocimiento del stock y de la especie, CLIMPROD escoge primero ajustar el modelo exponencial para la función $\mathbf{U}=\mathrm{f}(\mathbf{E}) \mathrm{y}$ encontrar $\mathrm{R}^{2}=86 \%$. La relación entre los residuales de este modelo y $\mathrm{V}$ es lineal (Fig. 7) y por lo tanto se ajusta el modelo lineal-exponencial y provee un valor de $\mathrm{R}^{2}$ igual a $95 \%$ (Fig. 8). La validaciónjackknife indica que todos los parámetros son significativos al nivel del $5 \%$ y que ningún año contribuye en más del $35 \%$ en cualquier estimación de coeficientes, lo que es relativamente satisfactorio. Los residuales del modelo no están autocorrelacionados.

\section{Pesquería de anchoveta del Pacifico suroriental}

Se reanalizan los datos presentados por Yáñez (1991) sobre la pesquería de anchoveta realizada por Perú y Chile entre 1957 y 1977. En este caso se considera que la variable ambiental que afecta la abundancia del stock es la temperatura superficial del mar (TSM) rezagada en 6 meses; es decir, estimada considerando los 6 primeros meses del año y los 6 últimos meses del año anterior.

Siguiendo las instrucciones de CLIMPROD se ajusta en primer lugar el modelo lineal para la función $\mathbf{C P U E}=\mathrm{f}(\mathbf{E})$ con un $\mathrm{R}^{2}=79 \%$. Luego, al considerar el efecto de la TSM, el sofware escoge ajustar el modelo lineal-lineal y provee un valor de $\mathrm{R}^{2}=91 \%$, el cual es validado por el método jackknife (Fig. 9). Se deduce que el stock de anchoveta del Pacífico suroriental es afectado por la variable ambiental, produciendo disminuciones en la producción cuando ésta aumenta (Fig. 10).

\section{DISCUSION}

Los resultados de los ejemplos de aplicación, presentados sólo para ilustrar las capacidades del software, no serán analizados con más detalle en este trabajo. El mayor interés es la discusión sobre las 
mejoras, limitaciones y riesgos que trae consigo la aproximación por el sistema-experto.

La introducción de una variable ambiental en los modelos globales de producción aumenta el número de parámetros en la formulación final y consecuentemente las siguientes principales dificultades: - Aunque el ajuste es más fácil, los límites de confianza de los parámetros son a menudo altos y el procedimiento de ajuste puede ser inestable.

- A veces es difícil estimar la contribución real de cada variable ( $\mathbf{E}$ y $\mathbf{V}$ ) en los modelos, debido a su interacción y/o colinearidad.

- El problema de los estados de transición se hace más difícil de resolver, especialmente cuando la influencia ambiental se describe por medio de una función de forma (en tales casos CLIMPROD no provee una solución satisfactoria).

- Aumentando el número de variables explicatorias también se aumenta la probabilidad de obtener buenas correlaciones aleatorias, independientemente de cualquier fenómeno biológico real (Ricker, 1975). La literatura provee muchos ejemplos de buenos ajustes históricos que se desmoronan en cuanto se usa el modelo para pronosticar.
Estas dificultades, comunes para cualquier regresión múltiple, pueden soslayarse seleccionando objetivamente las variables (sustentadas por observaciones biológicas en la medida de lo posible). Tal como lo han subrayado Bakun y Parrish (1980), la selección de la variable ambiental a introducir en el modelo, en lo posible, deber ser a priori y no sólo empírica (ellos presentan una lista de variables posibles).

Además, aquellos modelos aún tienen las limitaciones usuales de los modelos convencionales de excedentes de producción, asociadas a los supuestos básicos discutidos por Fox (1974). Aun después de su modificación, siguen siendo empíricos los procedimientos para evaluar las respuestas de los stock $\mathrm{s}$ de peces (en términos de biomasa y rendimiento) a los cambios de la tasa de pesca y de las condiciones ambientales. Por lo tanto, representan una aproximación a ciegas para investigar la variabilidad del reclutamiento.

Cuando no se pueden predecir los factores ambientales causales y/o procesos, y tienen un efecto de corto plazo, la aproximacción propuesta sólo puede servir para evaluar el rango de las variaciones

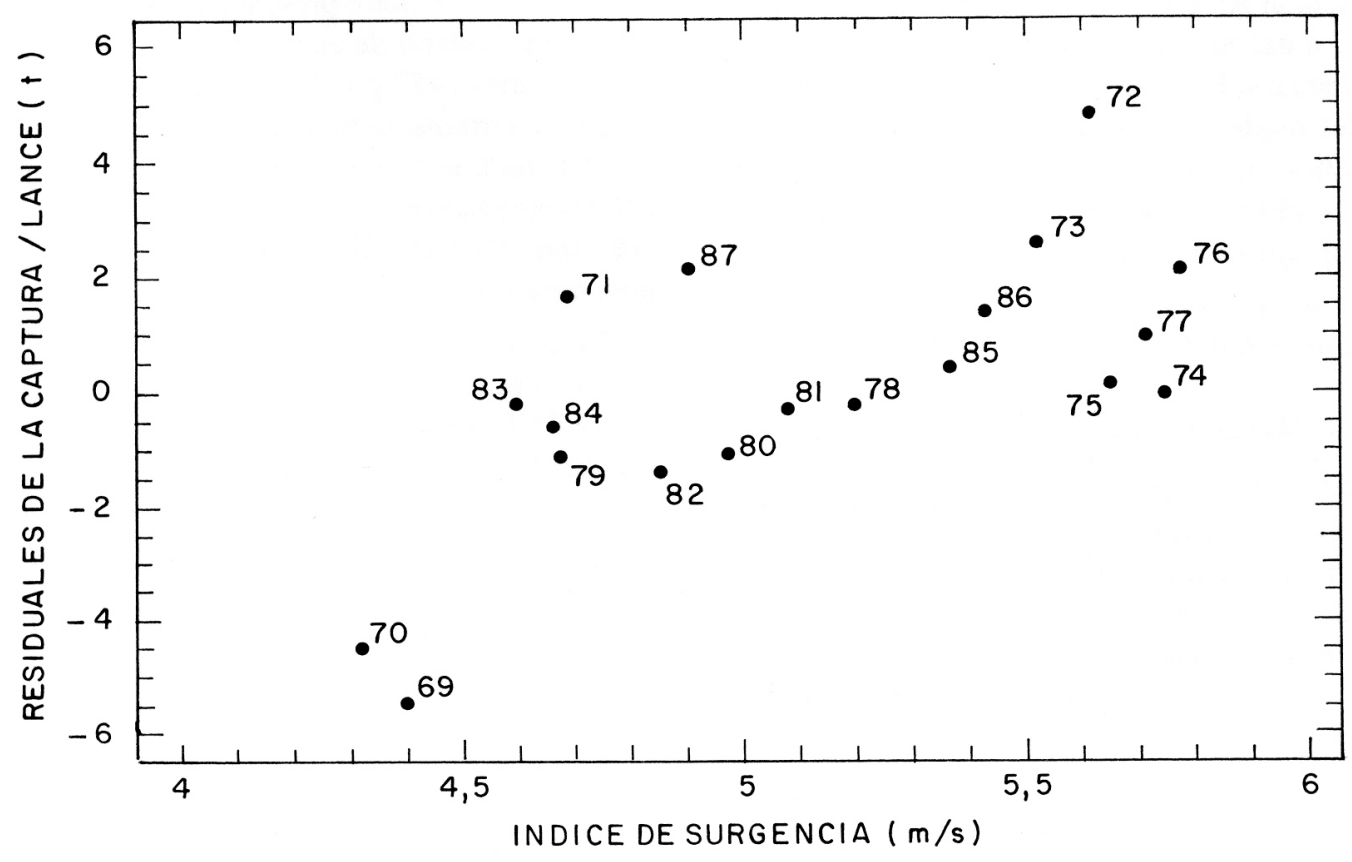

Figura 7. Gráfico de dispersión codificado entre el índice de surgencia (promedio ponderado de dos años de la velocidad del viento durante la época de surgencia) y los residuales de la captura por lance (de un modelo convencional de excedente de producción), donde los números representan años. 


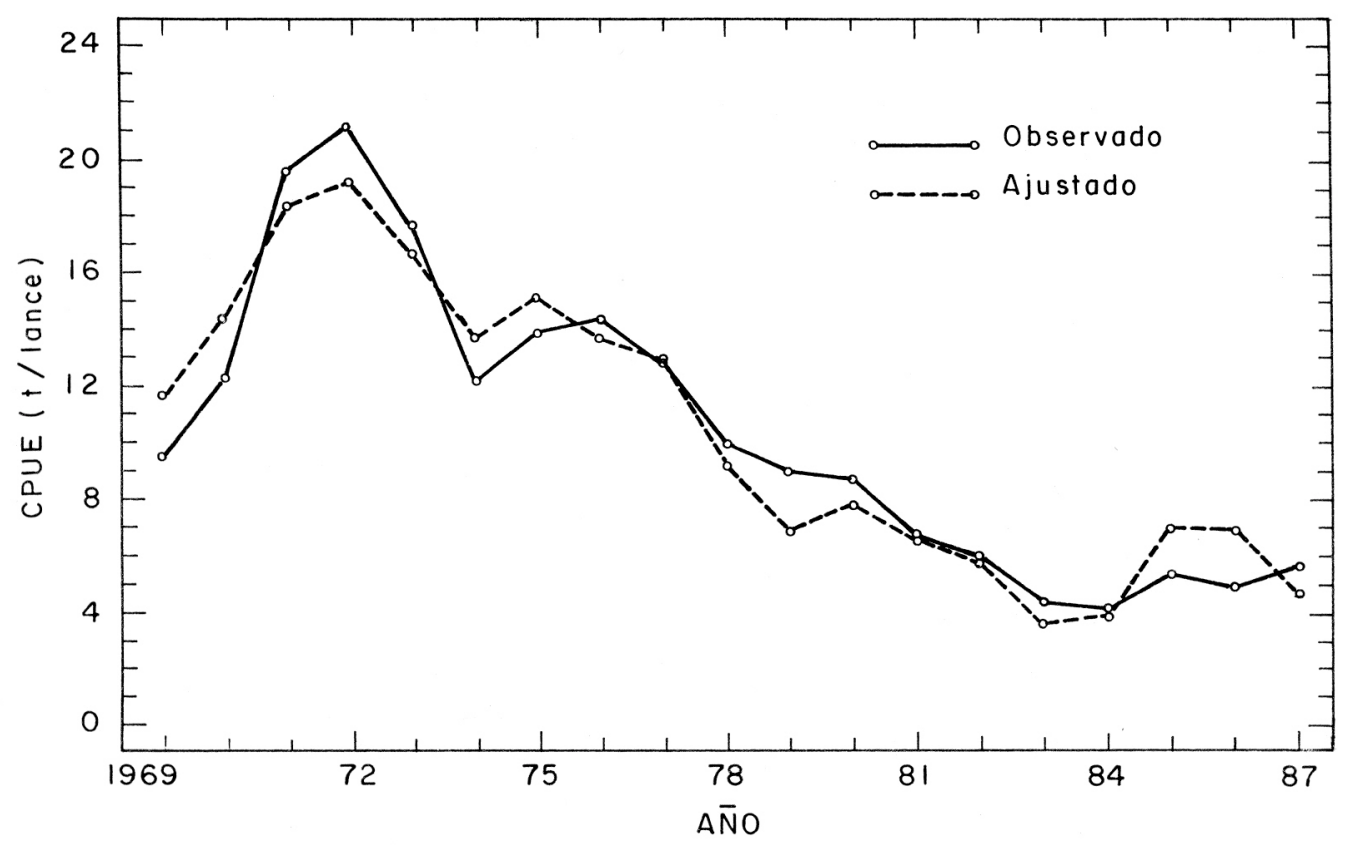

Figura 8. Capturas observadas de sardina por lance y ajustados considerando una variable ambiental en el modelo de excedente de producción.

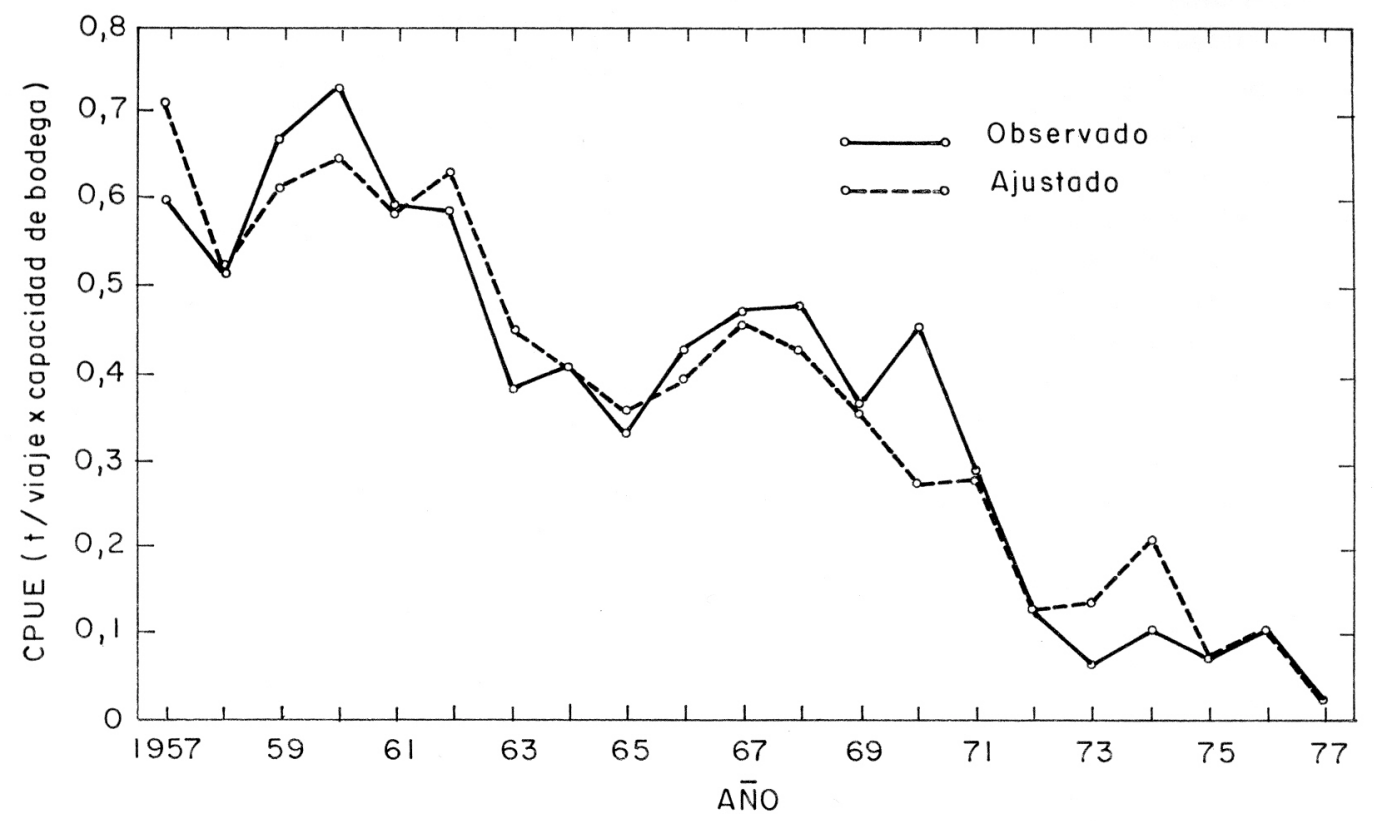

Figura 9. CPUE de anchoveta observadas y ajustadas al modelo lineal-lineal considerando el esfuerzo de pesca y la TSM como variable explicatorias. 


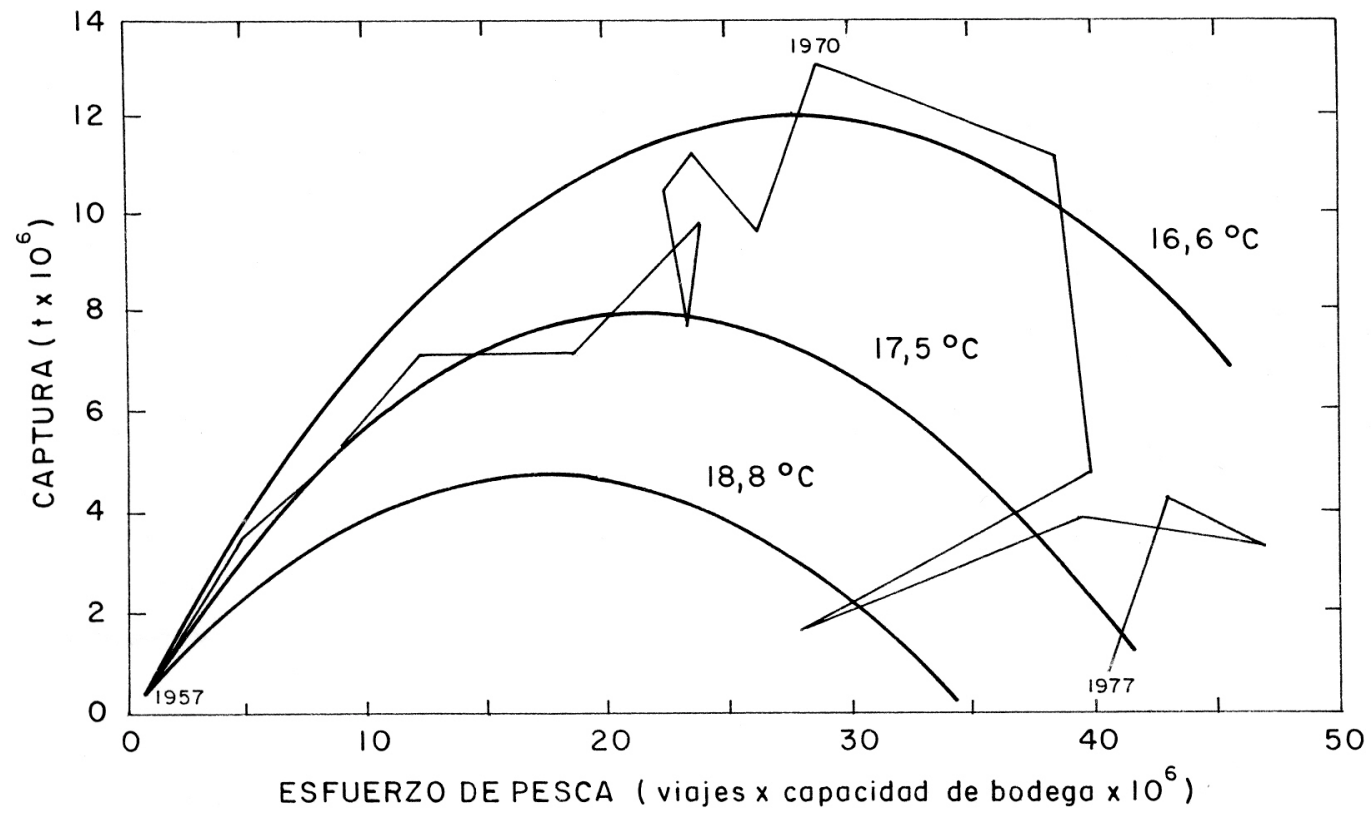

Figura 10. Modelo de producción lineal-lineal de anchoveta considerando tres valores de TSM.

ambientales inducidas y para compararlas con aquellas de los efectos de la pesca. Esto por sí solo sería útil, riores e inferiores de su biomasa y/o capturabilidad.

Los modelos simples de excedentes de producción han sido criticados porque sufren de falta de realismo biológico. No obstante, en muchas instancias más sofisticadas los modelos estructurados a la de edad, como los propuestos por Deriso (1980), no se comportan mejor debido a las dificultades en la estimación de los parámetros adicionales (Ludwig y Walters, 1985). CLIMPROD sólo usa una variable adicional y de cero a tres (pero a menudo un) parámetros adicionales a los modelos convencionales de excedentes de producción. Más aún, la inteligencia artificial permite usar datos cuantitativos o cualitativos adicionales que no están incluidos en el modelo como variables, pero que ayudan al usuario a escoger la mejor ecuación modelo de acuerdo a las características del stock y no sólo a los criterios de mejor ajuste. Se ha demostrado que estos criterios no necesariamente proveen políticas de prescripción más realistas (Uhler, 1980). La presente aproximación puede proveer una mejor evaluación y manejo del stock, al tomar en cuenta el conoci- miento de la biología o estructura del stock y la experiencia del experto con otros stocks.

Algunos aspectos negativos de CLIMPROD deben ser también señalados. Esta herramienta estarádisponible para biólogos-pesqueros o administradores de pesquerías y puede ser usada para ajustar cualquier modelo sin tener conocimientos especiales de dinámica de poblaciones. El programa hace que el usuario responda a varias preguntas con respecto a los supuestos básicos subyacentes del modelo y se detiene en caso de conocimientos insuficientes. No obstante, el usuario permanece libre y es responsable de otros errores. Más aún, la selección objetiva de una variable ambiental (incluyendo su ventana espacio-temporal y su retardo sobre la producción) es a menudo el factor clave para evitar correlaciones espúreas. En general, aún en el caso de los modelos de excedentes de producción, se requiere un conocimiento mínimo del stock y de la biología de la especie.

\section{CONCLUSIONES}

Pocos estudios sobre relaciones entre las especies marinas y su ambiente permiten una estimación de los efectos combinados del ambiente y del esfuerzo 
de pesca sobre el stock en términos de la producción efectiva y del MSY (maximun sustainable yield).

Los modelos delineados aquí permiten tomar en cuenta los efectos del ambiente sobre los rendimientos y por lo tanto soslayar las dificultades causadas por dos requerimientos subyacentes de los modelos convencionales de excedentes de producción, a saber: el set de datos debe referirse al período en que los factores ambientales que influyen sobre la abundancia del stock eran estables (o fluctuaron aleatoriamente sobre un período de observación suficientemente largo), en tanto que la capturabilidad también debe ser independiente del ambiente. Esta ventaja permite un aumento de la serie de datos utilizables, pero requiere más parámetros a estimar. La decisión sobre si usar los modelos tradicionales o sus versiones modificadas propuestas aquí, resultará del balance entre tales consideraciones.

Como estos modelos aún son globales, ellos retienen las limitaciones de tales modelos y requieren de otros supuestos básicos usuales. A pesar de tales restricciones, estos modelos son a menudo una solución más aceptable que los modelos tradicionales, especialmente en áreas tropicales donde los factores ambientales constituyen la influencia predominante sobre la producción de especies de vida corta. En tales áreas la determinación de la edad de los peces es frecuentemente difícil y requiere de muestreos intensivos y costosos, debido a la alta variabilidad de la longitud de los peces dentro de las cohortes asociadas con un tipo especial de agregación, como en el caso de especies pelágicas pequeñas (Fréon, 1985). Bajo tales circunstancias los métodos analíticos usuales son difícilmente utilizables. Aunque los modelos ambientales de producción no necesitan datos biológicos cuantitativos, es necesario poseer un conocimiento mínimo de la ecología de la especie para su correcta utilización. Uno de los propósitos del sistema-experto CLIMPROD es forzar al usuario a tomar en cuenta este conocimiento para seleccionar y ajustar un modelo apropiado. Esta experiencia en inteligencia artificial, a través del dialogo necesario entre el computador y las ciencias biológicas, conduce a la formulación de reglas de modelaje, las que a menudo son empíricas y crudas. Tal simplificación del modo de pensar del biólogo no está desprovista de interés. Por otra parte, el programa permite el intercambio de ideas entre expertos. Más aún, el software mis- mo podría ser una interesante herramienta pedagógica, ya sea cuando se usa con datos reales o con datos simulados.

La utilización de estos modelos para predicciones no está libre de riesgo. Esta requiere de un pronóstico del esfuerzo de pesca y en algunos casos del factor ambiental (cuando no hay un des fase suficiente entre este factor y su efecto sobre la pesquería). Este último pronóstico es a menudo impreciso, como lo señala Walters (1987). Más aún, los límites de confianza de los parámetros son a veces tan altos que las predicciones dentro del rango observado de las variables serían peligrosas y, por supuesto, sería aun peor pronosticar utilizando valores de entrada fuera del rango observado.

No obstante, el concepto del MSY, a pesar de su epitafio (Larkin, 1977), aún puede ser usado pero en un sentido amplio con los presentes modelos, los que pueden ser considerados como un desarrollo final. Ellos proveen diferentes MSY para cada estado .de la variable ambiental, o al menos un $\mathbf{f}_{\text {max }}$ diferente cuando sólo la capturabilidad es modificada. Los ejemplos de aplicación presentados sobre áreas de surgencia, entre otros (Fréon, 1988; 1991 b), muestran que los modelos CLIMPROD pueden proveer una interpretación bastante buena de la historia de la pesquería. Ellos pueden explicar cómo grandes fluctuaciones de las capturas (y algunas veces colapsos), pueden ocurrir sin ningún aumento del esfuerzo nominal, sino como resultado de cambios ambientales. Tal eventualidad ya ha sido prevista en los modelos estocásticos (Laurec et al., 1980), pero sólo de un modo estadístico. Aquí se propone una herramienta determinística para uso de los administradores de pesquerías. A pesar de la imprecisión en las predicciones de captura, permite comprender, y a veces pronosticar, tendencias de la pesquería. En este último caso, el objetivo no es sólo preservar el recurso, sino también optimizar la producción de excedentes dada por situaciones ambientales favorables.

\section{REFERENCIAS BIBLIOGRAFICAS}

Bakun, A., y R.M. Parrish. 1980. Environmental inputs to fishery population models for eastern boundary current regions. In: Workshop on the effects of environmental variation on the survival of larval pelagic fishes. G.D. Sharp (Ed.), Inter. 
Governmental Oceanographic Commission Rep., 28: 323 pp.

BIaxter, J.H. y J.R. Hunter. 1982. The biology of Clupeoids fishes. Adv. Mar. Biol., 20: 1-223.

Breiman, L. y J.H. Freidman. 1985. Estimating optimal transformation for multiple regression and correlation. J. Am. Statist. Ass., 80: 580-598.

Csirke, J. y G.D. Sharp. 1983. Reports of the expert consultation to examine changes in abundance and species composition of neritic fish resources. FAO Fish. Report, 291 (1): 102 pp.

Cury, P. y C. Roy. 1989. Optimal environmental window and pelagic fish recruitment success in upwelling areas. Can. 1. Fish. Aquat. Sci., 46: 670680

Deriso, R.B. 1980. Harvesting strategies and parameters estimation for an age-structured model. Can. J. Fish. Aquat. Sci., 37: 268-282.

Dickie, L.M. 1973. Interaction between fishery managment and environmental protection. J. Fish. Res. Board Can., 30: 2496-2506.

Dixon, W.J. y M.B. Brown. 1979. BMDP-79: biomedical computer programs. P. séries. University of California Press, Berkeley, CA, USA, $880 \mathrm{pp}$.

Doubleday, W.G. 1976. Environmental fIuctuations and fisheries managment. ICNAF Selected Papers, 1: 141-150.

Duncan, G.T. 1978. An empirical study of jacknifeconstructed: confidence regions in nonlinear regression. Technometrics, 20 (2) : 123-129.

Erron, B. y G. Gong. 1983. A leisurely look at the bootstrap, the jackknife and cross-validation. The American Statistician, 37 (1): 36-48.

Fletcher, R.I. 1978. On the restructuring of the Pella Tomlinson system. Fish. Bull., 76 (3): 515521.

Fréon, P. 1983. Production models as applied to substocks depending on upwelling fIuctuations. FAO Fish. Report, 291(3): 1047-1064.

Fréon, P. 1984. Des modèles de production appliqués à des fractions de stocks dépendantes des vents d'upwelling (pêche sardinière au Sénégal). Océanogr. Trop., 19 (1): 67-94.
Fréon, P. 1985. La variabilité des tailles individuelles a l'intérieur des cohortes et des bancs de poissons: 11. Application à la biologie des pêches. Océanol. Acta, 8 (1): 87-99.

Fréon, P. 1988. Introduction of environmental variables into global production models. In: Long term changes in marine fish populations. T. Wyatt and M.G. Larraneta (Eds.), Consejo Superior de Investigaciones Científicas, Vigo, España: 481-528.

Fréon, P. 1991a. Seasonal and interannual variations of mean catch per set in the Senegalese sardine fisheries: fish behaviour or fishing strategy? In: Long-term variability of pelagic fish populations ans their environment. T. Kawasaki, S. Tanaka, Y. Toba and A. Taniguchi (Eds). Pergamon Press: 135-145.

Fréon, P. 1991b. L'introduction d'une variable climatique dans les modèles globaux de production. In: Pêcheries ouest-africaines: variabilité, instabilité et changement. P. Cury et C. Roy (Eds.), ORSTOM, Paris: 395-424.

Fréon, P., C. Mullon y G. Pichon. 1993. CLIMPROD: Experimental interactive software for choosing and fitting surplus production models induding environmental variables. FAO Computerized Information Series (Fisheries), 5: $1-76$.

Fox, W.W. 1970. An exponential surplus-yield model for optimizing exploited fish populations. Trans. Am. Fish. Soc. 99 (1): 80-88.

Fox, W.W. 1971. Randon variability and parameters estimation for the generalized production model. Fish. Bull. (U.S.), 69 (3): 569-580.

Fox, W.W. 1974. An overview of production modeling. In: Workshop on tuna population dynamics. ICCAT. Rec. Doc. Scient., 3: 142-156.

Fox, W.W. 1975. Fitting the generalized stok production model by least squares and equilibrium approximation. Fish. Bull. (U.S.), 73 (1): 23-36.

Garrod, D.J. 1969. Empirical assessments of catch effort relationship in the North Atlantic cod stocks. Res. Bull. ICNAF, 6: 26-34.

Graham, M. 1935. Modern theory of exploiting a fishery and application to North Sea trawling. J. Cons. Explor. Mer, 10: 264-274.

Griffin, W.L., R.D. Lacewll y J.P. Nichols. 1976. 
Optimum effort and rent distribution in the Gulf of Mexico shrimp fishery. Amer. J. Agr. Econ.: 644652.

Gulland, J.A. 1969. Manual of methods for fish stock assessment. Part 1: Fish population analysis. FAO Man. Fish. Sci., 4: 154 pp.

Hilborn, R. y C.J. Walters. 1992. Quantitative fisheries stock assessment and management. Chapman and Hall, New York: 542 pp.

Laevastu, T. y H.A. Larkins. 1981. Marine fisheries ecosystems, its simulation and managment. Fishing News Book Ltd, Farnham, Surrey, England: 162 pp.

Laloé, F. 1989. Un modèle global avec quantité de biomasse inaccesible liée aux conditions environnementales: application aux données de la pêche ivoiro-ghanéenne de Sardinella aurita. Aquat. Living. Resour., 1: 289-298.

Larkin, P.A. 1977. An epitaph of the concept of maximum sustained yield. Trans. Am. Fish. Soc., 106(1): 1-11.

Lasker, R. 1985. What limits Clupeoids production?. Can. J. Aquat. Sci., 42: 31-38.

Laucee, A., A. Fonteneau y C. Champagnat. 1980. Etude de la stabilité des modeles autorégénérants, pp 423-438. In: The assessment and management of pelagic fish stocks. A. Saville (Ed.). Rapp. P.-v. Réun. Cons. int. Explor. Mer, 177: 517 pp.

Le Guen, J.C. y R. Chevalier. 1983. Etude des pêcheries: réflexions sur l' environnement et la gestion multispécifique. Rev. Trav. Inst. Pêches Marit., 46 (1): 9-70.

Loucks, R.H. y W.H. Sutcliffe. 1978. A simple fishpopulation model including environmental influence, for two western Atlantic shelf stocks. J. Fish. Res. Board Can., 35 (3): 279-285.

Ludwig, D. y C.J. WALTERS. 1985. Are agestructured models appropriate for catch-effort data? Can. J. Fish. Aquat. Sci., 42: 1066-1072.

Mae Arthur, A.H. y E.O. Wilson. 1967. The theory of island biogeography. Princeton Univ. Press, Princeton, N.J.: 203 pp.

Mac Call, A. 1984. Population models of habitat selection, with application to the northern anchovy. Nat. Mar. Fish. Serv., Southwest
Fischeries Center. Adm., Rep. LJ-84-01: 98 pp.

Marquardt, D.W. 1963. An algorithm fo leastsquare estimation of non linear parameters. J. Soc. Ind. Appli. Math., 11: 431-441.

Nelder, J.A. y R. Mead. 1965. A simplex method for function minimization. Comput. J., 7: 307313.

Nelson, W.R., M.C. Ingham y W.E. Shaff. 1977. Larval transport and year-class strength of atlantic menhaden, Brevoortia tyrannus. Fish Bull. (U.S.), 75 (1): 23-41.

Orbi, A., A. Agoumi, G. Ben Jannet y S. Tazi. 1991. Approche modélatrice des fluctuations d' abondance des sardines sur la côte nordatlantique marocaine. In: Pêcheries ouestafricaines: variabilité, instabilité et changement. P. Cury et C. Roy (Eds.), ORSTOM, Paris: 425-438.

Parrish, R.H. and A. Mac Call. 1978. Climatic variation and exploitation in the pacific mackerel fishery. Fish. Bull., 167: 110 pp.

Pella, J.J. y P.K. Tomlinson. 1969. A generalized stock production model. IATTC Bull., 13 (3): 419496.

Ricker, W.E. 1975. Computation and interpretation of biological statistics of fish populations. Bull. Fish. Res. Board Can., 191: 382 pp.

Rivard, D. y L.J. Bledso. 1978. Parameter estimation for the Pella-Tomlinson stock production model under non equilibrium conditions. Fish. Bull. U.S., 76 (3): 523-534.

Roff, D.A. y D.J. Fairbairn. 1980. An evaluation of Gulland's method for fitting the Schaefer model. Can. J. Fish. Aquat. Sci., 37: 1229-1235.

Saville, A. 1980. The assessment and management of pelagic fish stocks. Rapp. P.-V Réun. Cons. int. Explor. Mer, 177: 517 pp.

Schaefer, M.B. 1954. Some aspects of the dynamics of populations important to the management of the commercial marine fisheries. Bull. IATTC, 1 (2): $27-56$

Schaefer, M.B. 1957. A study of the dynamics of the fishery for yellowfin tuna in the eastern tropical Pacific ocean. Bull. IATTC, 1 (2): 245- 285. 
Schnute, J. 1977. Improved estimates from Schaefer production model: theoretical considerations. J. Fish. Res. Board Can., 34: 583-603.

Sharp, G. 1980. Workshop on the effects of environmental variation on the survival of larval pelagic fishes. IOC Workshop Report, 28: 321 pp.

Sharp, G.D. y J. Csirke. 1983. Proceedings of the expert consultation to examine changes in abundance and species composition of neretic fish resources. FAO Fish. Rep., 291 (2) and (3): 1-553 and 557-1224.

Silvert, W. 1983. Amplification of the environmental fIuctuations by marine ecosystems. Oceanologica Acta, $\mathrm{N}^{\circ}$ Special: 183-186.

Soutar, A. y J.D. Isaacs. 1974. Abundance of pelagic fish during the 19th and 20th centuries as recorded in anaerobic sediment offthe California. Fish. Bull. (U.S.), 72 (2): 257-273.

Steele, J.H. 1984. Kinds of variability and uncertainty affecting fisheries. In: Exploitation of marine communities. R.M. May (Ed.), Dahlem Konferenzen, Springer- Verlag, Berlin: 245-262.

Steele, J.H. y E.W. Henderson. 1984. Modeling longterm fIuctuations in fish stocks. Science, 224: 985987.

Troadec, J.P. 1982. Introduction à l' aménagement des pêcheries: interêt, difficultés, et principales méthodes. FAO Doc. Tech. Pêches, 224: 64 pp.
Uhler, R.S. 1980. Least squares regression estimates of the Schaefer production model: some Monte Carlo simulation results. Can. J. Fish. Aquat. Sci., 37: 1284-1292.

Walter, G.G. 1973. Delay differential equation models for fisheries. J. Fish. Res. Board Can., 30: 939-945.

Walter, G.G. 1975. Nonequilibrium regulation of fisheries. ICNAF Res. Doc., 75/IX/131.

Walter, G.G. 1986. A robust approach to equilibrium yield curves. Can. J. Fish. Aguat. Sci., 43: 12321239.

Walters, C.J. 1987. Nonstationarity of production relationships in exploited populations. Can. J. Fish. Aguat Sci., 44 (Suppl. 2): 156-165.

Yáñez, E. 1989. Fluctuaciones de los principales re. cursos pelágicos explotados en la zona norte de Chile y variaciones ambientales asociadas. Pacífico Sur, Número Especial: 509-520.

Yáñez, E. 1991. Relationships between environmental changes and fIuctuating major pelagic resources exploited in Chile (1950-88). In: Long-term variability of pelagic fish populations and their environment. T. Kawasaki, S. Tanaka, Y. Toba and A. Taniguchi (Eds). Pergamon Press: 301-309.

Yáñez, E., M.A. Barbieri y L. Santillán. 1992. Longterm environmental variability and pelagic fisheries in Talcahuano, Chile. In: Benguela trophic functioning. A.I.L. Payne, K.H. Brink, K.H. Mann and R. Hilborn (Eds.). S. Afr. J. mar. Sci., 12: 317.

Recibido el 8 de abril de 1994.

Aceptado el 21 de Julio de 1995. 


\section{Apéndice 1. Modelos disponibles en CLIMPROD}

Modelos $\mathbf{U}=\mathbf{f}(\mathbf{E})$

1. $U=$ a.exp(b.E)

2. $U=a+b \cdot E$

3. $U=(a+b \cdot E)^{(1 /(c-1))}$

Modelos $\mathbf{U}=\mathbf{f}(\mathbf{V})$
4. $U=a+b . V$
(lineal)
5. $\mathrm{U}=\mathrm{a} \cdot \mathrm{V}^{\mathrm{b}}$
6. $U=a+b \cdot V^{c}$
7. $U=a \cdot V+b \cdot V^{2}+c$
(exponencial)
(general)
(cuadrático)

(exponencial)
(lineal)
(generalizado)

Modelos $U$ = $\mathbf{f}(E, V)$; influencia de $V$ sobre la abundancia
8. $U=a \cdot V+b \cdot E$
(lineal-lineal)
9. $U=a+b . V+c . E$
(lineal-lineal)
10. $U=a \cdot V^{b}+c \cdot E$
(lineal-exponencial)
11. $U=a \cdot V+b \cdot V^{2}+c \cdot E$
(lineal-cuadrático)
12. $U=(a+b \cdot V) \cdot \exp (c \cdot E)$
(exponencial-lineal)
13. $U=a \cdot V \cdot \exp (b . E)$
(exponencial-lineal)
14. $U=a \cdot \exp (b \cdot E)+c . V+d$
(exponencial-lineal/aditivo)
15. $U=a \cdot V^{b} \cdot \exp (c . E)$ (exponencial-exponencial)
16. $U=a \cdot V^{b} \cdot \exp \left(c . V^{d} \cdot E\right)$ sin restricciones (exponencial-exponencial)
17. $U=\left(a . V+b . V^{2}\right) \cdot \exp (c . E)$ (exponencial-cuadrático)
18. $U=\left(\left(a . V^{b}\right)+c . E\right)^{(1 /(d-1))}$ (generalizado-exponencial)
19. $U=\left(\left(a+b \cdot V^{2}\right)^{(d-1)}+c \cdot E\right)^{(1 /(d-1))}$ (generalizado-cuadrático)

Modelos $U$ = f $(E, V)$; influencia de $V$ sobre la capturabilidad
20. $U=a \cdot V+b \cdot V^{2} \cdot E$
(lineal-lineal)
21. $U=a+b \cdot V-c \cdot(a+b \cdot V)^{2} . E$
(lineal-lineal)
22. $U=a \cdot V^{b}+c . V^{(2 . b)} \cdot E$
(lineal-exponencial)
23. $U=a \cdot V \cdot \exp (b . V . E)$
(exponencial-lineal)
24. $U=(a+b \cdot V) \cdot \exp (-c \cdot(a+b \cdot V) \cdot E)$
(exponencial-lineal)
25. $U=a \cdot V^{b} \cdot \exp \left(c \cdot E \cdot V^{b}\right)$
(exponencial-exponencial)
26. $U=a . V .(b-c . V)-d \cdot V^{2} \cdot(b-c \cdot V)^{2} \cdot E$
(lineal-cuadrático)
27. $U=a \cdot V \cdot(1+b \cdot V) \cdot \exp (c \cdot V \cdot(1+b \cdot V) \cdot E)$
(exponencial-cuadrático)

Modelos $U=f(E, V)$; influencia de $V$ sobre ambos abundancia y capturabilidad
28. $U=a \cdot V^{(b+c)}+d \cdot V^{(2 \cdot b)} \cdot E$
(lineal-exponencial-exponencial)
29. $U=a \cdot V^{(1+b)}+c \cdot V^{(2+b)}+d \cdot V^{(2 \cdot b)} \cdot E$
(lineal-cuadrático-exponencial)
30. $U=a . V^{b} . \exp \left(E . c . V^{d}\right)$ con restriccion de signo (exponencial-exponencial-exponencial)
31. $U=\left(a \cdot V^{(1+b)}+c \cdot V^{(2+b)}\right) \cdot \exp \left(d \cdot V^{b} \cdot E\right)$ (exponencial-cuadrático-exponencial) 